\title{
From the Lexicon to Expectations About Kinds: A Role for Associative Learning
}

\author{
Eliana Colunga \\ University of Colorado at Boulder
}

\author{
Linda B. Smith \\ Indiana University Bloomington
}

\begin{abstract}
In the novel noun generalization task, 2 1/2-year-old children display generalized expectations about how solid and nonsolid things are named, extending names for never-before-encountered solids by shape and for never-before-encountered nonsolids by material. This distinction between solids and nonsolids has been interpreted in terms of an ontological distinction between objects and substances. Nine simulations and behavioral experiments tested the hypothesis that these expectations arise from the correlations characterizing early learned noun categories. In the simulation studies, connectionist networks were trained on noun vocabularies modeled after those of children. These networks formed generalized expectations about solids and nonsolids that match children's performances in the novel noun generalization task in the very different languages of English and Japanese. The simulations also generate new predictions supported by new experiments with children. Implications are discussed in terms of children's development of distinctions between kinds of categories and in terms of the nature of this knowledge.
\end{abstract}

Concepts are hypothetical constructs, theoretical devices hypothesized to explain data, what people do, and what people say. The question of whether a particular theory can explain children's concepts is therefore semantically strange because strictly speaking this question asks about an explanation of an explanation. We begin with this reminder because the goal of the research reported here is to understand the role of associative processes in children's systematic attention to the shape of solid things and to the material of nonsolid things in the task of forming new lexical categories. These attentional biases have been interpreted in terms of children's concepts about the ontological kinds of object and substance (e.g., Dickinson, 1988; Imai \& Gentner, 1997; Soja, Carey, \& Spelke, 1992; Subrahmanyam, Landau, \& Gelman, 1999). These concepts and the notion of a psychological ontology are theoretical constructs offered within the framework that posits propositional representations.

In the simulations and experiments reported here, we show how abstract distinctions about different kinds of categories may be made through associative learning and the patterns of correlations between the perceptual properties of things and words. The theoretical explanation we offer and the experiments we report do not

Eliana Colunga, Department of Psychology, University of Colorado at Boulder; Linda B. Smith, Department of Psychology, Indiana University Bloomington.

This research was supported by National Institutes of Health-National Institute of Mental Health Grant R01 MH60200 and by National Institutes of Health-National Institute of Child Health and Human Development Grant R01 28675 to Linda B. Smith. We thank Michael Gasser for assistance with the modeling and collection of data from Japanesespeaking adults, Larissa Samuelson for discussions of the simulations and for making available the judgments from English-speaking adults, and Hanako Yoshida for assistance with the Japanese data collection and discussion of the conceptual issues.

Correspondence concerning this article should be addressed to Eliana Colunga, Department of Psychology, University of Colorado, Boulder 345 UCB, Boulder, CO 80309-0345. E-mail: colunga@psych.colorado.edu explain the hypothetical constructs of object and substance nor do they fully replace these constructs by explaining all of the data that have been subsumed under them. Nonetheless, the results do show how simple associative processes may create abstract distinctions about different kinds and, in so doing, these processes may play a crucial role in children's category and lexical learning.

\section{The Hypothesis}

There is no doubt that associative mechanisms are part of children's psychology; however, there is considerable debate about the role of these processes in early lexical learning, with most of the discussion centered on whether associative processes are sufficient to explain all of children's progress in this domain (e.g., Bloom, 2000; Golinkoff et al., 2000; Hollich et al., 2000; Jones, Smith, \& Landau, 1991). It seems highly unlikely that associative learning can explain it all, but it also seems highly likely that these general learning processes make important contributions to children's developing knowledge about lexical categories.

One particular strength of associative learning is pattern matching, that is, internalizing the structure of the learning environment. This is sometimes seen as a limitation, with the criticism being that associative mechanisms output pretty much what is put in (e.g., Keil, 1994). This limitation, however, may be a strength in the larger context of development. Developmental change occurs over a series of encounters with the environment. What one takes from each of these encounters depends on what one already knows. By internalizing the regularities across previous encounters, associative learning may create expectations that guide and constrain future learning. In this way, associative learning may be a powerful bootstrap on learning itself.

One way these internalized regularities may guide future learning is by influencing attention. In laboratory learning experiments, it has been shown that properties that systematically co-occur in the learning environment direct attention to each other (e.g., Murphy \& Wisniewski, 1989; Wattenmaker, 1991, 1993). A second way that associative learning may bootstrap more advanced learn- 
ing mechanisms is through the discovery of higher order generalizations. In any reasonably complex environment, there are regularities at several levels of abstraction (see McRae, de Sa, \& Seidenberg, 1997; Yoshida \& Smith, 2003b). If an associative learning device can discover the higher order regularities, then it can form rulelike generalizations that transcend the specific properties of specific instances. These rulelike generalizations, in turn, may characterize the nature of fundamentally different kinds.

We pursued these ideas by concentrating on children's performances in a widely used experimental task known as the novel noun generalization task. The skills that $21 / 2$-year-olds show in this task are remarkable precisely because the task itself provides so little information and support. In the typical experiment, a child is shown a single object and told its name. From that information alone, the child is asked to indicate the category to which the name applies by extending the name to other things. Moreover, the typical experiment uses stimuli that are novel made-up things labeled with novel made-up names such that there is little information about the category to be formed. Nonetheless, with just this little to go on, 2- to 3-year-old children form categories for solids and nonsolids differently. When they are shown a single novel solid thing and told its name, 2- to 3-year-olds generalize that name to things that match the original in shape. But when they are shown a single novel nonsolid thing and told its name, same-age children often extend the name to new instances that match in material (Colunga \& Smith, 2003; Imai \& Gentner, 1997; Soja et al., 1992).

Other research has shown that children can use a variety of kinds of information to guide category formation-information about function, about how the stimuli were made, and about roles in complex events (e.g., Bloom, 1996; Booth \& Waxman, 2002a, 2002b; Diesendruck, Markson, \& Bloom, 2003; Gathercole, Cramer, Somerville, \& Haar, 1995; Gelman \& Bloom, 2000; Kemler Nelson, Frankenfield, Morris, \& Blair, 2000). However, the evidence from the novel noun generalization task shows that 2- to 3 -year-old learners do not need this information; rather, quite minimal stimulus cues about solidity appear to activate knowledge about different kinds and do so in a way that generalizes to never-before-encountered things.

Several additional facts about this phenomenon are also relevant. First, what the experimenter says in the task critically determines children's performance. Children adaptively shift their attention to different properties for different kinds when the experimenter names (e.g., Landau, Smith, \& Jones, 1988) the objects or uses language associated with talking about kinds (e.g., "another," "same kind"; e.g., Diesendruck \& Bloom, 2003). Children do not systematically shift attention to different dimensions when they are asked to group objects or to make similarity judgments. This indicates that it is not the stimulus properties alone that determine children's attention to different properties. Rather, the language that defines the task also matters. Second, children younger than 2 years do not so clearly differentiate solids and nonsolids, even in explicit naming tasks (Samuelson, 2002; Samuelson \& Smith, 1999). Moreover, the degree to which individual children show these kind-specific shifts in attention appears to be strongly related to the number of nouns known by that child, as if children learn the predictive relation between solidity and category structure as they learn early nouns (Samuelson, 2002; Samuelson
\& Smith, 1999; Yoshida \& Smith, in press). In this article, we report evidence pertinent to this hypothesis.

We propose the following: First, the early noun lexicon presents regularities between solidity, nonsolidity, and shape- versus material-based categories. Second, through general processes of associative learning, children internalize these regularities. Third, these internalized regularities form a higher level distinction about solids and nonsolids as different kinds. This last prediction, that these associations create abstract knowledge about different kinds, brings us close to the idea that children's performance in this task is related to their concepts of object and substance. We reserve discussion of this issue to the General Discussion, concentrating here and in the experiments on the data to be explained-children's attention to the shapes of solids and the materials of nonsolids in the novel noun generalization task.

\section{Background}

There is considerable evidence that the developmental roots of these context-specific attentional shifts begin well before word learning. Infants discriminate among perceptual cues associated with solidity and nonsolidity (E. J. Gibson, Owsley, Walker, \& Megaw-Nyce, 1979; Mash, Quinn, Dobson, \& Narter, 1998; Spelke, Breinlinger, Macomber, \& Jacobson, 1992). Moreover, Huntley-Fenner, Carey, and Solimando (2002) showed that 8-month-old infants attend to the number of cohesive (typically solid) things but do not attend to the number of noncohesive (typically nonsolid) things. These results indicate that infants not only are sensitive to the relevant perceptual cues for distinguishing solids and nonsolids but also know something about their meaningful correlations. Apparently, however, these early sensitivities are not enough to drive kind-specific attention in the novel noun generalization task because well-differentiated attentional biases for solids and nonsolids emerge only well after children have learned some names for solid and nonsolid things (e.g., Samuelson \& Smith, 1999). This last fact raises the possibility that the different attentional biases for solids and nonsolids that are typically shown by 2 - to 3 -year-olds in the novel noun generalization task are a consequence of linking early perceptual sensitivities to words.

If this possibility is so, this developmental work also begins in infancy. In one important study, Waxman and Markow (1995) examined 12-month-old infants' ability to form categories in a familiarization paradigm. They presented infants with instances of the category animal (e.g., bear, duck, lion, and dog) during a familiarization phase and with novel within-category or out-ofcategory instances during the test phase (e.g., cat vs. apple). A novelty preference for the out-of-category instance was interpreted as evidence of categorization. The central result was that infants showed this novelty preference only when the original instances had been named during familiarization, a result that suggests that naming directs attention to category-relevant properties.

In recent studies, Booth and Waxman (2003) suggested further that by 14 months, children know something quite specific about the linguistic cues in English that are associated with noun categories; they found that infants attend to object categories only given a word presented as a novel count noun (e.g., "a dax") but not when presented as a novel adjective (e.g., "a daxy one"). These results provide clear evidence of early learned links between 
linguistic cues and kind of lexical category. The knowledge shown by infants in these tasks appears to be limited in two ways relative to that of 2- to 3-year-olds. First, there is no evidence to date that infants differentiate noun categories into different kinds for solids and nonsolids. Second, the experimental demonstrations with infants involve familiar categories and often presentations of multiple instances of the category. This is in contrast to older children's attentional shifts that are evoked by the naming of a single novel thing. Generalized expectations that differentiate different kinds and apply broadly to unfamiliar things apparently develop later in word learning.

Studies using the standard form of the novel noun generalization task-naming a single novel thing with a novel name-with older infants also have suggested progressive development toward more abstract expectations about how nouns map to categories. One key study is by Woodward, Markman, and Fitzsimmons (1994). They presented 13- and 18-month-old infants with a novel object, a strainer, and named it with a novel name ("this is a__"). They then tested the children's learning in two comprehension tasks: one in which the target object was identical to the original exemplar and one in which the target object was from the same adult lexical category (also a strainer) and highly similar overall to the exemplar (different in color). In three out of four experiments, the 13-montholds systematically mapped the name to the identical object but not to the merely highly similar object. The 18 -month-olds, in contrast, more consistently mapped the name to the identical object and to the highly similar object. These results fit the idea that children do not initially know the full range of instances that fall within noun categories.

By 24 months of age, there is evidence of this sort of knowledge for solid things but not so clearly for nonsolid things (e.g., Imai \& Gentner, 1997; Kobayashi, 1997; Landau et al., 1988; Samuelson \& Smith, 1999; Soja, Carey, \& Spelke, 1991; Subrahmanyam et al., 1999). Given a single novel solid exemplar and told its name, 2-year-olds reliably extend that name to instances that match in shape, even when these shape-matching instances differ dramatically from the exemplar in other properties (e.g., Samuelson \& Smith, 1999). The material bias for nonsolids is much weaker and often not reliable at this age. In some studies 2-year-olds have been reported to extend names for nonsolids by material (Soja, 1992; Soja et al., 1991, 1992), but in others they have not, either overgeneralizing the shape bias for solids to nonsolids (Samuelson, 2002; Samuelson \& Smith, 1999; Subrahmanyam et al., 1999) or responding at chance levels (Imai \& Gentner, 1997). All in all, the evidence indicates that children's knowledge of the predictive relation between solidity and category structure becomes stronger between 2 and 3 years of age.

Other evidence indicates that children's name extensions in these tasks are influenced by syntactic cues, specifically those concerning the noun's status as count or mass (e.g., Dickinson, 1988; McPherson, 1991; Soja, 1992). In English, count nouns are preceded by $a$ or an and refer to discrete entities that can be counted (e.g., "a cup" or "an airplane"), and mass nouns are often preceded by some or much and refer to continuous masses that cannot be counted (e.g., "some water" or "much cheese"). Older children (3- and 4-year-olds) typically name solids with count nouns but often name nonsolids with mass nouns (see Gordon, 1988; Hall, 1994; Levy, 1988). In novel noun generalization tasks, count-noun syntax increases attention to shape, and mass-noun syntax increases attention to material, although these syntax effects are stronger for nonsolids than solids and for older than younger children (Soja, 1992; Subrahmanyam et al., 1999). Mass-count syntax is a property of English but not a property of all languages. These syntax effects thus raise the question of whether different languages - by offering perhaps different patterns of correlations with shape- and material-based categories-lead to different developmental outcomes.

Cross-linguistic studies have found both similarities and differences in children learning different languages, including languages that differ in ways one may think should matter (Colunga \& Smith, 2004; Gathercole, 1997; Imai \& Gentner, 1997). For example, both children learning English (which has mass-count syntax) and children learning Japanese (which does not have mass-count syntax) attend to the shapes of solids more than nonsolids in the novel noun generalization task (Imai \& Gentner, 1997). However, there have also been reports of cross-linguistic differences. In one study, Imai and Gentner found that whereas 2 1/2-year-old English-speaking children generalized names for solids formed into simple shapes by shape, same-age Japanese-speaking children showed no clear preference, generalizing names for simply shaped solids sometimes by material and sometimes by shape. Imai and Gentner also found that the material bias for nonsolids appeared earlier and was more robust for Japanese-speaking children than for English-speaking children (see also Kobayashi, 1997; Samuelson \& Smith, 2000). There is even evidence that language may have long-term effects; adult speakers of Japanese and Yucatec Mayan (a language with material-based nouns) are more likely to attend to material than are adult speakers of English (Imai \& Gentner, 1997; Lucy \& Gaskins, 2001).

To summarize, the developmental evidence suggests (a) an early sensitivity to the properties that distinguish solids and nonsolids; (b) an early sensitivity to words as indicators of categories; (c) incremental progress toward increasingly generalized expectations about how nouns map to differently structured categories for solids and nonsolids, with knowledge about solids developing earlier than knowledge about nonsolids; and (d) a possible role for language learning in this developmental trend. In light of this evidence, we propose that associative learning is the mechanism that takes infants' discriminations of solids and nonsolids, their sensitivity to words, and their sensitivity to the structures of familiar categories and transforms them into generalized expectations about how even novel solid and nonsolid things are named. We suggest further that learning names for specific solid and nonsolid things is the critically relevant experience. In other words, our proposal is that 2- to 3-year-old children's performances in the novel noun generalization task are the product of generalizations over the regularities that characterize the nouns they already know. By this account, children may not show well-differentiated categories of novel solids and nonsolids earlier because doing so requires having already learned a noun corpus that reliably shows these regularities.

Given this proposal, there are a number of testable predictions that one might make. In what follows, we concentrate on predictions that derive from two key ideas. First, the statistical regularities that characterize the nouns that 2- and 3-year-old children typically know should strongly predict these children's category formations in the novel noun generalization task. Second, the mathematical function that predicts children's performances from 
the statistical regularities in the early noun corpus should describe well-accepted ideas about associative learning and generalization by similarity.

\section{Structure of the Lexicon}

Samuelson and Smith (1999) reported evidence pertinent to these predictions. They studied the regularities that characterize the corpus of nouns on the Macarthur Communicative Developmental Inventory (MCDI; Fenson et al., 1993). The MCDI is a parent checklist of the early words and phrases commonly produced by 18 - to 30 -month-old children. The checklist was developed from extensive studies of parental diaries, in-laboratory testing of early vocabularies, and large normative studies of the instrument itself (Fenson et al., 1993). More specifically, the words included on the MCDI are those known by $50 \%$ of children at 30 months. Thus, the 312 nouns that are on the MCDI represent a typical noun lexicon for 2- to 3-year-old children learning English.

Samuelson and Smith (1999) examined the statistical regularities presented by the categories in this corpus through the use of a method pioneered by Rosch (1973a, 1973b). Adults were presented with each noun in the corpus and asked to think of common instances of the category. They were then asked a series of "yes/ no" questions about the instances named by the noun: Are these similar in shape? Are these similar in color? Are these similar in material? Are these solid? Are these nonsolid? A separate group of adults was presented with criteria for distinguishing count and mass nouns and asked to judge whether each noun on the MCDI was a count noun, was a mass noun, or could be used in both syntactic frames (e.g., cake). From these adult judgments, each lexical item was designated as shape-based, color-based, materialbased, based on none of these properties, or based on any combination of these properties. Each noun was also designated as referring to solid or nonsolid things and as a count or mass noun. Individual nouns were designated as having these properties if $85 \%$ of the adult judges agreed with the designation.

Figure 1 summarizes the key regularities in terms of Venn diagrams. In these diagrams, the relative size of each circle rep- resents the relative number of nouns of that kind, and the size of the overlap between intersecting circles represents the relative number of nouns of both kinds. The circles in the left panel depict the relative numbers of count nouns, names for solid things, and names for categories organized by shape. The circles in the right panel represent the relative numbers of mass nouns, names for nonsolid substances, and names for things in categories organized by material. What Figure 1 shows is that many early nouns are count nouns, many refer to solid objects, and many name objects in shape-based categories. Moreover, count nouns, solid things, and shape similarity go together, albeit imperfectly. The right panel in Figure 1 shows that there are many fewer nouns in this corpus that are mass nouns, name nonsolid things, and name categories organized by material. Further, nonsolidity, mass-noun syntax, and material-based categories seem to go together, again, however, imperfectly. Figure 1 also shows that there are many exceptions to these regularities.

Figure 1 could be interpreted as indicating that the early noun lexicon presents the relevant regularities and thus is a potential source for children's expectations about how solid and nonsolid things are named. Alternatively, if one focuses on the exceptions, one might conclude that the relevant regularities are too weak to account for children's knowledge. One way to determine whether the statistical properties of the early noun lexicon could possibly create children's generalized expectations is to present those regularities to an associative learning device and see just what is learned. This was one of the main goals of the simulation studies that follow.

There are, in addition to the regularities studied by Samuelson and Smith (1999), properties of solid and nonsolid things that must be taken into account in any attempt to model the correlations presented by early noun categories. Most critical, solids and nonsolids differ in the kinds of shapes they can take. Solid things can be a variety of shapes, from broken, irregular pieces to highly constructed and multipart forms with right angles and parallel sides. Nonsolid things, in contrast, can take a much more restricted range of shapes-flatter, rounder, and less constructed. Moreover, nonsolid things in the world of children-for example, oatmeal,
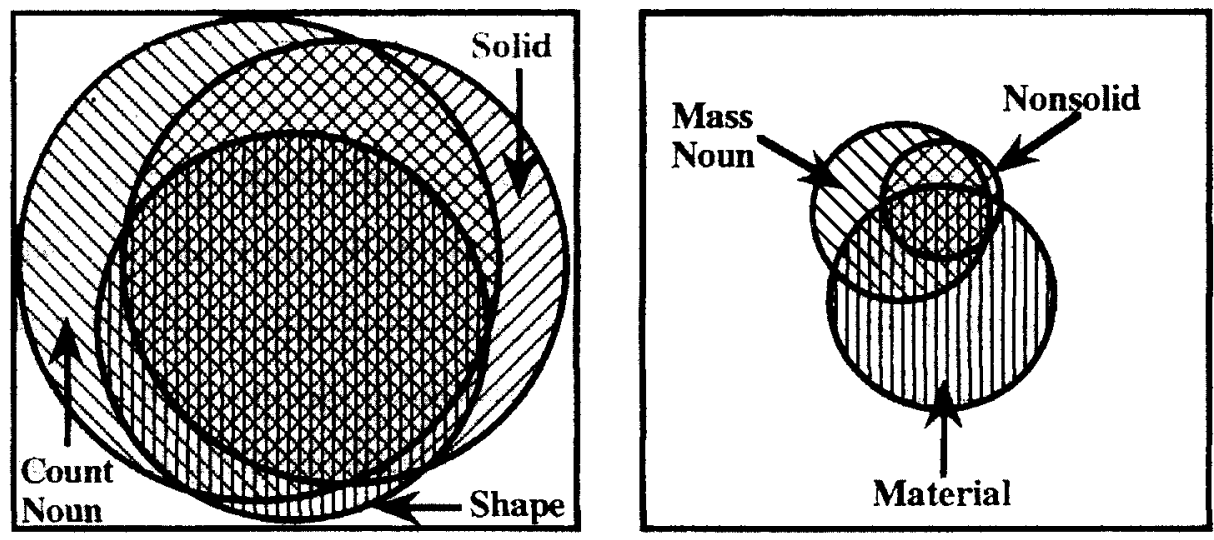

Figure 1. Regularities in the early English lexicon, presented as Venn diagrams. Size of area indicates the number of words for categories of solid things (left) and nonsolid things (right). Reprinted from Cognition, 73, L. Samuelson and L. B. Smith, "Early Noun Vocabularies: Do Ontology, Category Structure and Syntax Correspond?" p. 11. Copyright 1999, with permission from Elsevier. 
juice, and applesauce-hold their shapes for at best very brief periods of time and often transform into the accidental shapes of splatters and spills. All of this is relevant because shapes with right angles and parallel sides suggest a "designed" form whereas shapes without these properties-for example, the splatter of oatmeal on the floor-appear accidental (see Biederman, 1987; Burger \& Prasada 1997; Leyton, 1992; Prasada, Ferenz, \& Haskell, 2002). Further, Gelman and Bloom (2000) showed that both adults and children use cues about whether shapes are designed or not to make inferences about the relevance of shape to object categories. Recall, also, that Imai and Gentner (1997) found that shape complexity affected 2 1/2-year-old Japanese-speaking children's performances in the novel noun generalization task. Thus, in addition to the lexical category regularities studied by Samuelson and Smith, there are regularities generated by physics-solid things can hold highly complex shapes whereas nonsolid things cannot-and there is also evidence that these correlations may matter to children. Accordingly, we took these regularities into account in our simulations and provide evidence for their importance in the experiments with children as well.

\section{The Learning Problem}

Could an associative learner given sensitivities to the shape, material, and solidity and also given training on the nouns that label these things induce generalized expectations about how solid and nonsolid things are named? One possible limit on an affirmative answer to this question is the strength of the regularities themselves. They may be too noisy, characterized by too many exceptions, to generate the kind-specific generalizations that 2- to 3 -year-olds show. A second possible limit concerns associative learning mechanisms and whether an associative learner-even given near perfect correlations in the noun lexicon-could develop attentional biases of the form showed by children. From an associative learning perspective, children's generalized and kindspecific attentional biases must arise from learning specific associations between individual words and individual instances, from, for example, the pairing of a particular yellow ball with the word ball and the pairing of the orange juice one is drinking from a bottle with the word juice. But 2- to 3-year-olds' expectations about category structure are not expectations about specific things, or even about specific categories such as ball or juice. Rather, they are higher order expectations about how different kinds of categories are organized.

Knowing to categorize novel solid things by shape and novel nonsolid things by material requires the learner to make a secondorder generalization (Smith, Colunga, \& Yoshida, 2003) or overhypothesis (Goodman, 1955/1983; Shipley, 1993, 2000) that transcends particular objects, particular shapes, and particular categories. The critical correlation is not between cues, such as being solid and being chair-shaped, but between cues and category organization, between being solid and being in a category that is organized by shape-whatever that shape may be-and between being nonsolid and being in a category organized by materialwhatever that material may be. Can these kinds of overhypotheses emerge through general processes of associative learning, processes that consist of the pairings between names and instances? This is the key learning problem addressed in the simulation studies that follow.
A second learning problem concerns the graded and contextsensitive nature of the correlations in the learning environment and whether children's knowledge is likewise graded and contextsensitive. The performances of 2- to 3-year-olds in the novel noun generalization task seem rulelike; solids are named by shape, and nonsolids are named by material. But associative learners at best mimic rules (Marcus, 2001; Thornton, 2000). Thus, if children's expectations about how solid and nonsolid things derive from learned associations, then children should show the signature markings of frequency dependency and context dependency. More specifically, there should be a match between the statistical regularities that characterize the nouns that children know and the regularities that characterize their performances when they are generalizing a just-heard novel noun to new instances.

\section{Rationale}

In the simulations and experiments that follow, we tested the idea that 2- to 3-year-old children's novel noun generalizations are, at least in part, the product of the correlations that characterize already learned nouns and also general processes of associative learning. We provide three kinds of evidence. First, we show that an artificial associative learner (a connectionist network), when presented with the same regularities that characterize early nouns, develops generalized kind-specific attentional biases that are applied to never-before-experienced things, a shape bias for solids and a material bias for nonsolids. This evidence demonstrates the plausibility of the hypothesized mechanism. Second, we use the connectionist network to discover additional regularities in the learning environment, making and testing new predictions about children's novel noun generalizations. In this way, we show that the associative learning account is generative and has predictive power. Finally, we show that this account offers a unified explanation of both the overarching distinction between solids and nonsolids and the history- and context-sensitive nature of children's performances by applying the account to cross-linguistic data showing both similarities and differences in the novel noun generalizations of English- and Japanesespeaking children.

We specifically concentrate on the nouns known by 2- to 3-yearolds and (with the exception of one experiment) on the performances of 2- to 3-year-olds in the novel noun generalization task. We did this not because we believe this age is somehow special in the developmental trajectory or because we believe that children's generalized expectations about solids and nonsolids appear all of a sudden at this age. We did so because the literature suggests that kind-specific attentional shifts in the novel noun generalization task are reliable at this age. Thus, we can strongly predict that the nouns that 2- to 3-year-olds typically know should show the requisite regularities. In taking this methodological approach, we did not assume that the first 50 or first 100 or first 200 nouns are absent of the relevant correlations. Nor did we assume that signatures of early learned correlations might not be evident in younger children. Rather, we concentrate on the match between the statistical properties of the nouns known by 2- to 3-year-olds and the novel noun generalizations of children this age as a first step in examining the possible role of associative learning in creating expectations about the lexical category structure of different kinds. 
Before we proceed, it is also useful to make clear several additional untested assumptions and some assumptions that are not being made. It is critical that we assumed that children's cognitive processes include associative learning and generalization by similarity, processes we modeled with a Hebbian learning algorithm. We did not assume that these are the only processes with which children are endowed or that these processes can necessarily explain all aspects of children's cognition beyond their expectations in the novel noun generalization task. Nor did we assume that the specific instantiation of associative learning that we used in the simulations would be an entirely accurate model of even children's associative learning. However, we chose the particular modeling approach we did to make the most minimal and uncontroversial assumptions about the learning process. Thus, a more complete model of children's associative learning and of their cognition in general is likely to include the processes used here.

We also assumed that children begin word learning with welldeveloped sensitivities to the relevant perceptual and linguistic cues - that is, to properties such as solidity, nonsolidity, and the constructedness or accidental quality of shapes and to words as segmented units. There is considerable evidence for these assumptions (E. J. Gibson et al., 1979; J. J. Gibson, 1979; Huntley-Fenner et al., 2002; Mash et al., 1998; Spelke et al., 1992; Spelke \& Hespos, 2001); accordingly, our connectionist model incorporates these already developed abilities (see Roy \& Pentland, 2002, for a related approach). The critical claim of the model is not that lexical learning creates sensitivities to the properties of solidity and nonsolidity, or to the dimensions of shape and material, but rather that lexical learning creates a corpus of correlations between words, properties, and the dimensions that organize categories and that it is the internalization of these regularities that creates children's generalized expectations about categories of even novel solid and nonsolid things.

In addition, although we assumed that the nouns typically known by 2- to 3-year-olds present the relevant correlations, we made no assumptions about why children learn these nouns rather than other nouns. Past research has suggested that the nouns children know strongly reflect the nouns they hear (Huttenlocher, Haight, Bryk, Seltzer, \& Lyons, 1991) and that the nouns children hear present the same statistical regularities as the names they know (Sandhofer, Smith, \& Luo, 2001). Thus, the regularities we examined are likely to be those that characterize the learning environment. However, our assumptions are about the words children know; a corpus of lexical categories must be learned before the correlations within that lexicon can matter. In the General Discussion, we return to the question of why the early nouns that children learn have the structure that they do.

Finally, the simulations model correlational learning and children's generalizations from already learned nouns. The model was not designed to describe how children learned those original nouns in the first place. That is, the procedure we used to teach the network a corpus of nouns (the pairings of names and specific instances) does not adequately model the full complexity of the learning environment and the learning process but does, we believe, adequately model the correlational structure that is part of that learning environment and that is, by hypothesis, the basis of children's generalized expectations about the category structures of solid versus nonsolid things.

\section{Generalized Expectations From Learned Associations}

\section{Experiment 1}

The goal of this first experiment was to show that a simple associative learner, taught names for individual things, can learn a shape bias for categorizing solids and a material bias for categorizing nonsolids. This is a necessary first step because the ability of ordinary networks to solve this task is not obvious. Connectionist networks are simple associative learners, but the generalization required to yield a solidity-nonsolidity distinction like that evident in 2- to 3-year-olds is not simple because it requires going from simple associations between names and specific instances to abstract, rulelike generalizations. To do this, the learner-network or child-must go beyond specific experience at (at least) two levels of abstraction.

The first level of abstraction or first-order generalization is one readily made by associative devices and involves generalizing learned responses to new instances. For example, from the word ball associated with several specific round things of variable color and material and from the word sand associated with several specific heaps of a particular material and range of colors, the learner may generalize the name ball to other similarly round things and the name sand to similar materials. These first-order generalizations are typically easy for networks as they readily learn correlational patterns among properties and labels (e.g., Christiansen \& Chater, 2001; Farkas \& Li, 2001; Moss, Hare, Day, \& Tyler, 1994; Regier \& Carlson, 2001; Schyns, 1991).

It is the second level of abstraction that gives rise to what can be called overhypotheses and that is less clearly in the repertoire of simple associative networks. These higher order correlations concern the relevant dimension-the importance of shape in general, for example, rather than the importance of particular shapes. Moreover, children's generalizations reflect not one but two higher order correlational patterns: Children seem to know that solidity signals the relevance of shape and nonsolidity signals the relevance of material, even for objects and substances never encountered before and shapes and materials never experienced before. The ability of ordinary associative networks to simultaneously learn and generalize two such patterns has not been well studied. In a prior effort, Samuelson (2002) attempted to teach a similar network both a shape and a material bias, but it appeared to be capable of learning only one at a time. Samuelson attempted to model a particular training regimen, used to teach shape and material biases to very young children, children too young to show a shape or material bias on their own. The children, like the network, failed to learn two biases and acquired only the shape bias. Samuelson's inability to teach the network two contextdependent biases could reflect the training regimen or it could reflect a more general inability of this kind of network to simultaneously form two context-dependent overhypotheses- that shape similarity matters for solids but that material similarity matters for nonsolids.

Accordingly, the goal of the first experiment was to investigate the ability of a simple Hebbian network to form these higher level correlations. A Hebbian network learns by strengthening connection weights between units activated at the same time. Given this form of learning, the higher level correlations that seem to underlie children's novel noun generalizations would have to emerge from 
the specific correlations among individual instances and their labels. The goal of the first experiment was to determine whether this learning was possible, given the best possible evidence that solid things are named by shape but nonsolid things are named by material.

Specifically, in this first experiment, the network was presented with a vocabulary that was unlike the one that children learn in that it perfectly reflected the hypothesized regularity: Half of the training categories were organized by shape, and half were organized by material; all of the shape-based categories consisted of solid things, and all of the material-based categories consisted of nonsolid things. If a simple Hebbian network categorizes novel things by shape in the context of solidity but categorizes novel things by material in the context of nonsolidity after learning this "perfect" training set, then we will have demonstrated that, in principle, children's preferences in the novel noun generalization task could result from learning names for specific instances.

\section{Method}

Architecture. We used a simple settling network, a variant of a Hopfield net. The network was trained using Contrastive Hebbian Learning (Movellan, 1990), an algorithm that adjusts weights on the basis of correlations between unit activations (see Appendix A). Figure 2 shows the architecture of the network. The network has a word layer in which words are represented symbolically, that is, locally: Each unit in the word layer corresponds to one word.

Individual entities are represented on the perceptual layer. Activation patterns on this layer represent the solidity, shape, and material of each individual object or substance presented to the network. More specifically, the shape and the material of an object (say the roundness of a particular ball and its yellow rubbery material) are represented by an activation

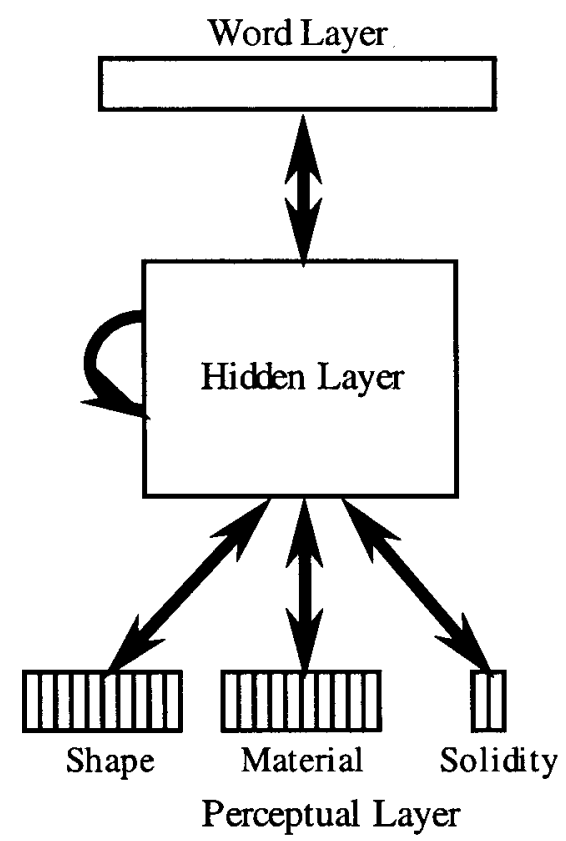

Figure 2. The perceptual layer represents the shape, material, and solidity of things. These are connected to the word layer through a recurrent hidden layer. Although not indicated in the figure, the perceptual layer is also recurrently connected to itself, as is the word layer. pattern along the whole layer, in a distributed fashion. Representing the perceptual properties of things in a distributed manner captures the graded similarities of things within and between categories and in so doing enables generalization by similarity. Solidity is represented locally; there is one unit that stands for solid and another unit that stands for nonsolid. We represent solidity categorically to capture the idea of what we take to be the main perceptual contrast - that of the stability of shape when touched (solid) versus the instability of shape (nonsolidity). The word layer is connected recurrently to itself as is the perceptual layer.

Finally, there is a hidden layer that is connected to all of the other layers and recurrently with itself. The word layer and the perceptual layer are connected only through the hidden layer; there are no direct connections among them. It is this last fact that makes this network different from a standard Hopfield net. We chose this approach of an intervening hidden layer so that the network could build internal representations that stand between the instances (solid and nonsolid things) and the nouns that label them. We were particularly interested in how these representations change as a consequence of learning a vocabulary in which solidity predicts shape-based categories and nonsolidity predicts material-based categories.

Overview of simulations. The rationale behind the simulations is as follows: First, we teach the network a vocabulary, in this case one that perfectly represents a correlation between solid and shape-based and between nonsolid and material-based. We teach the network a vocabulary by pairing "names" with individual "perceptual" patterns. Second, we test how the network categorizes novel things. That is, after learning this vocabulary, does the network "know" to attend to the shape of a novel solid thing but to attend to the material of a novel nonsolid thing?

Training. The training set for the network consisted of 20 lexical categories. Half of these words, the solid-shape words, were paired with categories instantiating a solid-shape-based correlation, and the other half, the nonsolid-material words, were paired with categories instantiating a nonsolid-material-based correlation. That is, each of the 10 solid-shape words was paired with individual instances of a category of things that were solid and shared a value along the shape dimension, and each of the 10 nonsolid-material words was paired with individual instances of a category of things that were nonsolid and shared a value along the material dimension. During an epoch, the network was trained on each of the words in the training set, paired with a randomly generated instance of that category. Thus, the network saw as many different instances of each word as the number of training epochs.

During training, a presentation of a word-instance pairing for the network consisted of the simultaneous activation of a unit on the word layer, a unit on the solidity layer, and shape and material patterns on the perceptual layer. The specific training patterns used in this simulation were generated in the following way. To make a solid-shape word, a unique unit in the word layer (name of the category) and the unit representing solid were activated together with a 12-bit binary number that was randomly generated to represent the shape of the object, the core of the category because it is a shape-based category. The value along the material dimension was left unspecified, to be determined randomly by the program as it trained the network on each instance of the category. Thus, during each presentation of a solid-shape word, the network learns associations among the unit in the word layer, the unit representing solid, a particular shape pattern, and variable material patterns. Examples of four instances of one solid-shape word and of one nonsolid-material word are shown in Figure 3.

Patterns for nonsolid-material words were generated analogously. These patterns consisted of a unique unit in the word layer, the unit representing nonsolid, a particular material pattern, and variable shape patterns. Thus, during training, the network was presented with many instances of each word, but the instances of a given solid-shape word always shared the same unique shape and had different materials, whereas the instances of a particular nonsolid-material word always shared the same unique material and had different shapes. 
a) Solid-Shape

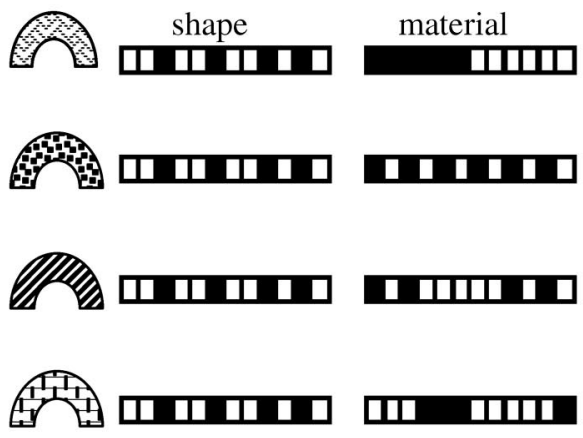

b) Solid-Material

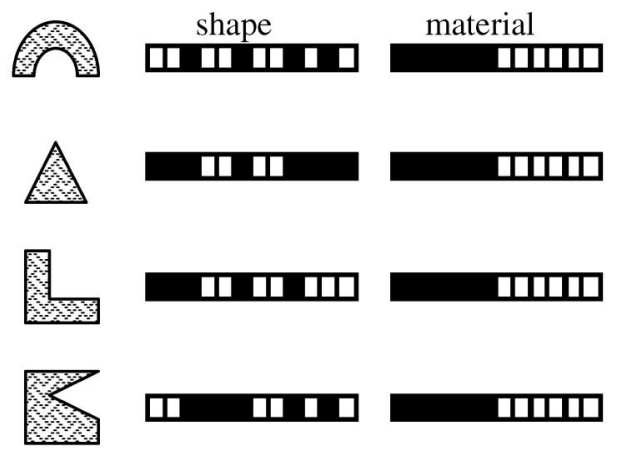

Figure 3. Examples of patterns of instances for (a) one shape-based category and (b) one material-based category. The activation pattern on the perceptual layer is represented by the shading of the units.

The networks were trained for 100 epochs, so over the complete training session, they were presented with a total of 100 instances for each of the 20 words in the training set. This duration of training was set on the a priori assumption that by the age of $21 / 2$ years, children have seen many instances of common categories of solid and nonsolid things.

Testing. After learning a training vocabulary that presents a perfect correlation between solidity and naming by shape and between nonsolidity and naming by material, does the network "know" to give a greater weight to the shape than to the material of a novel solid thing but to give a greater weight to the material than to the shape of a novel nonsolid thing? We addressed this question by presenting the network with novel input patterns - novel shapes and materials - and examining the resulting patterns of activations on the hidden layer. If the network has learned to highlight information about shape in the context of solidity, then the pattern of activation on the hidden layer given an input pattern marked as solid should represent mostly the shape information from the input pattern and not the material information. If, in addition, the network has learned to highlight information about material in the context of nonsolidity, then the pattern of activation on the hidden layer given an input pattern marked as nonsolid should represent mostly the material information from the input pattern and not the shape information. Thus, the patterns of activation on the hidden layer for two solid things of the same shape but different material should be highly similar. In contrast, the patterns of activation on the hidden layer for two nonsolid things of the same material but different shape should be highly similar (Smith, 1995; Smith, Gasser, \& Sandhofer, 1997).

Accordingly, each test trial consisted of presenting three input patterns - an exemplar, a shape match, and a material match — to the network and recording the activation pattern on the hidden layer after each of these patterns was presented and the network had settled. We call this pattern after settling the internal representation (IR) vector. The shape and material matches used in testing were generated by selecting the pattern along the matched dimension in the exemplar (e.g., shape for the shape match, material for the material match) and combining it with a different randomly generated pattern along the other dimension (e.g., variable material patterns for the shape match, variable shape patterns for the material match).

The structure of a test trial is shown in Figure 4. We first presented the network with an input pattern corresponding to a novel exemplar and then recorded the IR vector. Then we presented the network with an input pattern that presented the same shape but a different material (a shape match) and with an input pattern that presented the same material but a different shape (a material match), recording the IR vectors for both. Finally, we calculated the similarity between the IR vector for the novel exemplar and the IR vector for its shape match and between the IR vector for the novel exemplar and the IR vector for its material match. We used the absolute value of the difference between the vectors as a measure of similarity. If the network has learned the higher order correlations, then the IR vectors for the exemplar and the shape match should be more similar when they are solid and the IR vectors for the exemplar and the material match should be more similar when they are nonsolid. We simulated choices in a noun extension task by computing forced-choice probability using these similarity measures and Luce's choice rule (Rumelhart \& McClelland, 1986). The similarity measure (the absolute difference between the two vectors) was normalized by subtracting each difference value from the maximum difference found in the simulations. As per Luce (1963), the probability of choosing a particular test object was computed by dividing the similarity of the IR vectors for that choice by the sum of the similarity of the IR vectors for that choice and for the alternative choice. That is, the probability of a shape choice was calculated as the similarity of the IR vectors of the shape-matching test object divided by the sum of the similarities of the IR vectors for the shape-matching and materialmatching test objects.

The network was tested on 40 novel exemplars, each with four shape matches and four material matches, for a total of 160 test trials. Half of these trials involved patterns of activation representing solid things and half patterns representing nonsolid things. The 40 novel exemplar patterns used in testing were randomly generated and thus stand in no systematic relation to the training exemplars. However, both the training and test sets were randomly generated, and both sampled the entire space of possible instances. As Marcus (2001) noted, a training set that covers the whole space of possible instances may be crucial to enabling connectionist networks to form broad generalizations.

Ten networks were trained (with 10 different randomly generated initial connection weights) as described above. Appendix A provides the equations for the simulations, and Appendix B summarizes the architecture, training and testing sets, and the critical comparisons at test for this and for the remaining simulations in this article.

\section{Results and Discussion}

The networks readily learned the words in the training vocabulary. Figure 5 shows the networks' performance during training, that is, their ability to correctly label new instances of the trained categories. More specifically, Figure 5 shows the percentage of instances for which the correct unit in the word layer was activated as a function of number of passes (epochs) through the training set. The networks reached ceiling performance after 20 passes through the training vocabulary. Thus, in subsequent simulations, the networks were trained for only 32 epochs. 


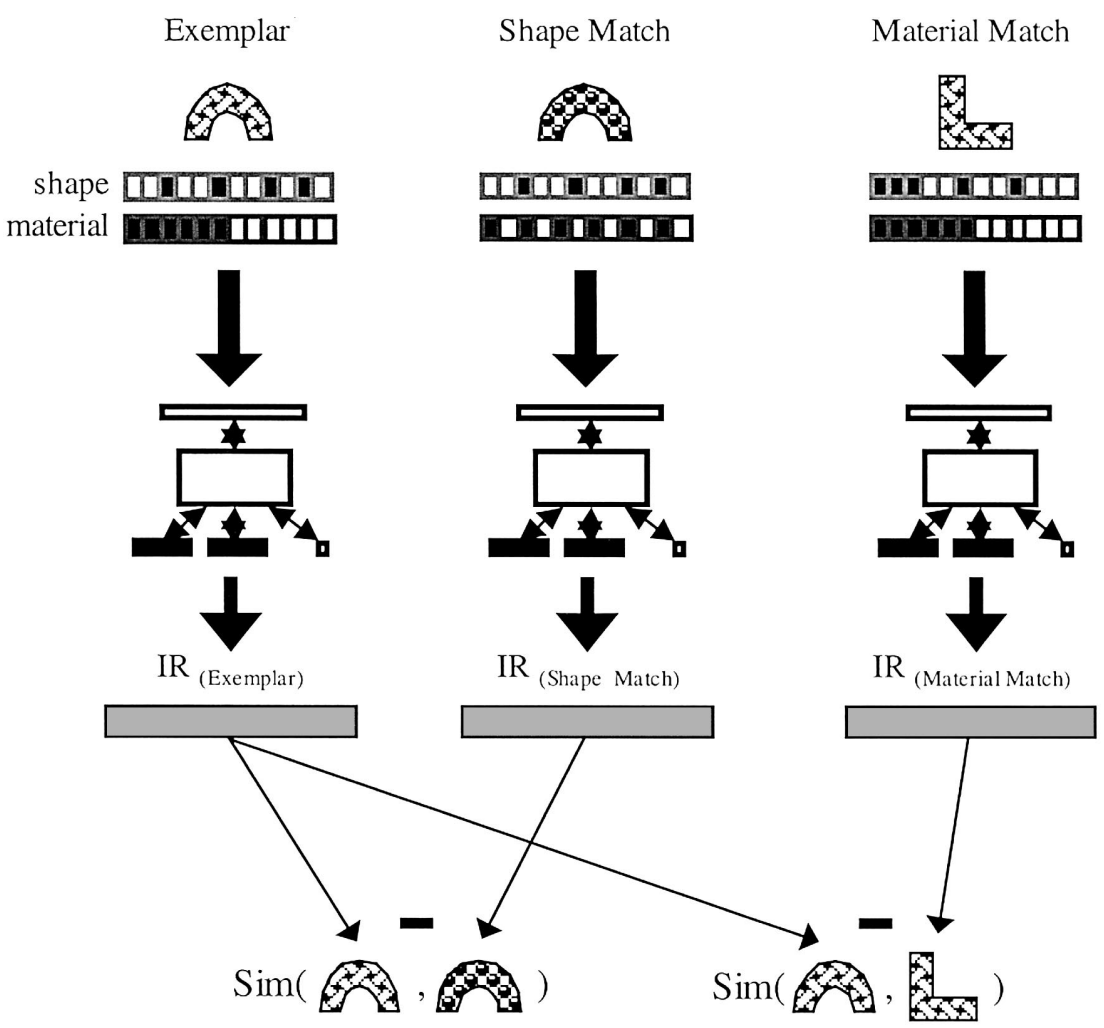

Figure 4. Structure of a network's test trial. The similarity (Sim) between the internal representations (IRs) of the target pattern and its shape and material matches is used to predict probability of choice.

Figure 6 shows the proportion of shape choices in the simulated version of the novel noun generalization task before and after training. Before training, the networks were unbiased; the difference between the proportion of shape choices for solids and nonsolids was not significant, $t(9)=0.086, p>.9$, and the proportions of shape choices predicted for both solid and nonsolid trials were not different from chance, $t(9)=-1.385, p=.2$, for solids; $t(9)=-1.022, p=.3$, for nonsolids

After training, the networks preferred the shape match for the solid exemplars and the material match for the nonsolid exemplars.

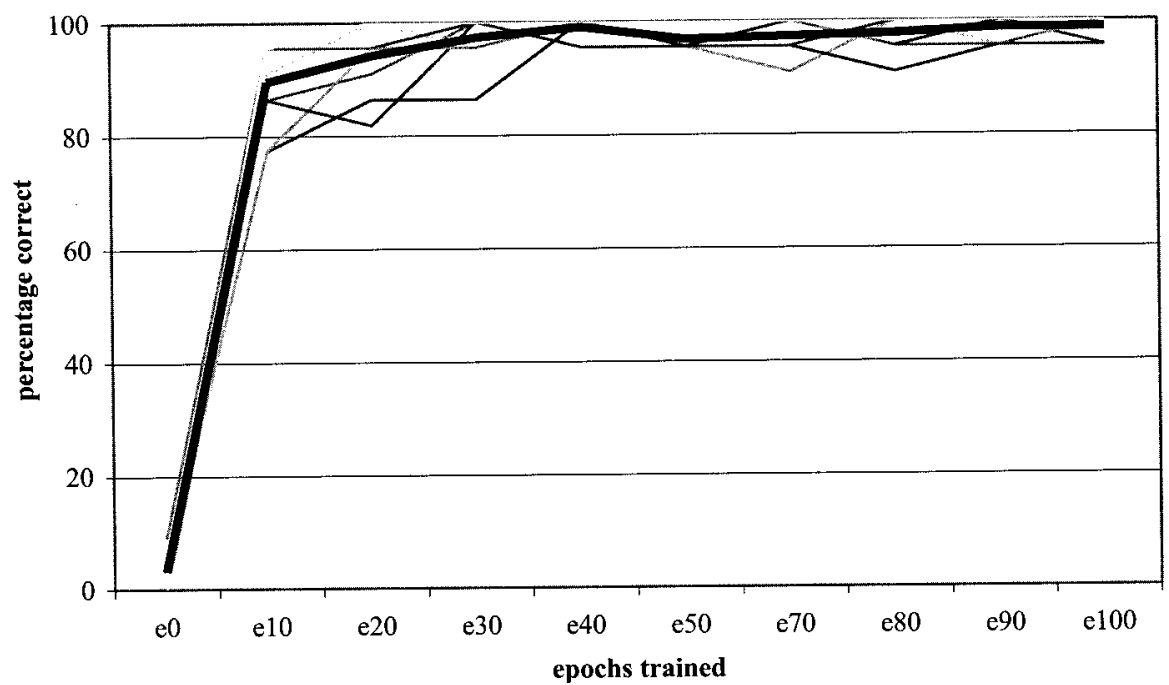

Figure 5. Correct productions of the target word given a training instance during the training phase for the 10 networks. The thick line represents the average performance of the 10 networks. $\mathrm{e}=$ epoch. 


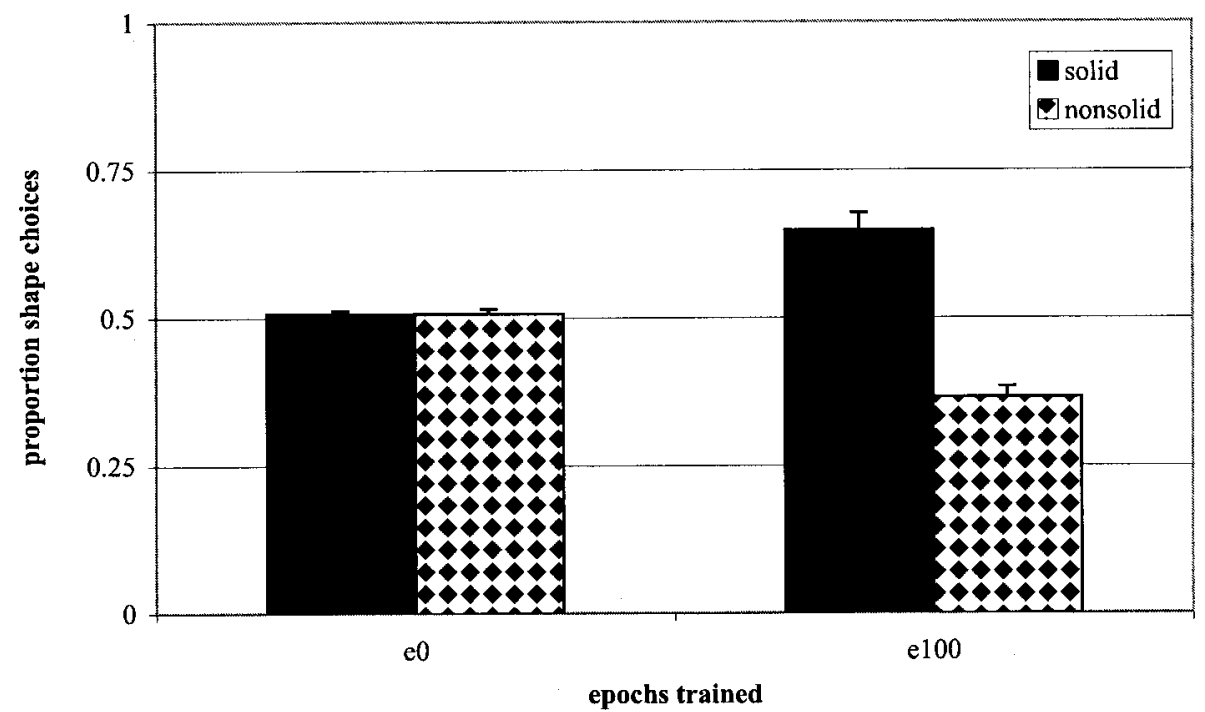

Figure 6. Mean proportion choices of the shape-matching test item by the networks for solid and nonsolid patterns prior to training $(\mathrm{e} 0)$ and after training (e100). Error bars represent standard error. e $=$ epoch.

A paired $t$ test comparing the proportion of shape choices predicted by the networks in solid trials versus nonsolid trials revealed that, after training, the difference was significant, $t(9)=12.105, p$ $<.0001$. In addition, the proportion of shape choices predicted for the solid trials was significantly greater than chance, $t(9)=5.425$, $p<.001$, and for the nonsolid trials was significantly less than chance, $t(9)=-8.170, p<.0001$. There was no such differential treatment for solids versus nonsolids in the networks before training. Eight of the 10 networks showed this pattern of shape choices for the solids and material choices for the nonsolids ( 2 of the networks developed one of the biases but not the other). These networks after training look like "idealized" children, with a strong shape bias for solids and a strong material bias for nonsolids.

It is critical that in these networks we know the processes that cause the different patterns of generalizations for solids and nonsolids. The different treatment of solid and nonsolid things results from changes in the way the network represents solid and nonsolid inputs. For solid inputs, shape is emphasized in the network's internal representations, so the network's representations of two patterns for solids that have the same shape but different material are more similar to each other than are its representations of two patterns for solids of the same material but different shape. The opposite is true for nonsolid inputs: The network's representations of nonsolids of the same material but different shape are more similar to each other than are the representations of nonsolids of the same shape but different material.

Figure 7 shows the effect of training on the network's representations as the average distance (absolute difference) between IR vectors for patterns representing solid things of the same shape, solid things of the same material, nonsolid things of the same shape, and nonsolid things of the same material. In the network's internal representations, solid things of the same shape but different material are more similar to one another than are solid things of the same material but different shape; nonsolid things of the same material but different shape are more similar to each other than are nonsolids of the same shape but different material. Thus, a connectionist network that is taught only associations between specific words and specific instances can show performance describable as knowledge of a more abstract kind if the associations present the higher order correlation: Solid things with the same name have the same shape, and nonsolid things with the same name have the same material. This result suggests that, at least in principle, it is possible to create a generalized distinction about different kinds through associative learning. As far as we know, this is the first demonstration that simple connectionist networks can acquire context-specific second-order generalizations.

One potential criticism of this demonstration is that the training sets were described to the network in terms of the potentially relevant properties - shape, material, and solidity. The inclusion of these properties is justified by developmental evidence on infant's sensitivities to them. However, the input to the network did not describe instances on a variety of other potentially irrelevant properties (e.g., size, color, or location). Thus, the network was presented with an easier task than children face. Of course, this is true on many grounds. All modeling is simplification. We offer two arguments to support the simplification of describing the inputs in terms of only the relevant properties. First, connectionist networks are adept pattern learners, well able to find the predictive relations and ignore the nonpredictive ones (see Thornton, 2000). To demonstrate this adeptness in the present case, we replicated the simulations but added irrelevant variation, specifying each input in terms of shape, material, "size," and "location" such that the one relevant property (shape for solids, material for nonsolids) comprised only $25 \%$ of the input pattern. In this set of simulations, the shape and material vectors were generated as described in the main simulations. The size and location vectors were randomly generated for each input and thus did not consistently predict category membership for solids or nonsolids. The results of this simulation were nearly identical to those reported above. Most important, the networks still showed a generalized bias to represent the shapes of solids but the materials of nonsolids. This replication, of course, does not prove the point as one could imagine schemes 


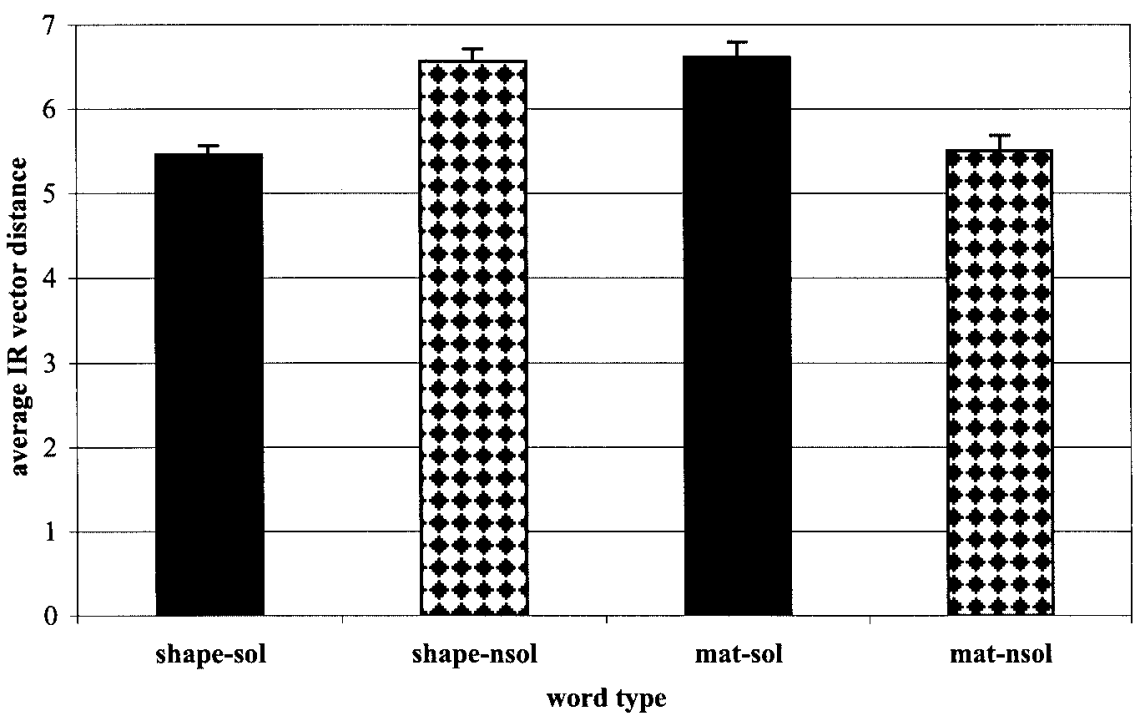

Figure 7. Raw similarity measures (absolute difference) for the internal representations (IRs) in Experiment 1. Error bars represent standard error. sol $=$ solid; nsol $=$ nonsolid; mat $=$ material.

in which the relevant properties comprise a vanishingly small proportion of the input vector. However, given the ability of associative engines to find predictive relations in noisy data, we suspect such networks would still be successful. Second, and more important in our view, theoretical descriptions of the training instances that emphasize solidity, nonsolidity, shape, and material seem justified in terms of the developmental evidence that strongly indicates children's perceptual systems are highly sensitive to these properties even before word learning (see, e.g., Bushnell, 1982; Bushnell \& Boudreau, 1991; Mash et al., 1998). Children do not begin word learning with an infinite list of possible dimensions but rather-through their biology and through their interactions with the world-have perceptual systems tuned to particular dimensions.

\section{Experiment 2}

Experiment 1 demonstrated that a simple associative learning device can form abstract generalizations about how different kinds are named. Or to be more specific, the networks can develop connection weights that emphasize shape in the context of a solidity cue but emphasize material in the context of a nonsolidity cue. We propose, however, that by taking seriously the details of the correlations in children's noun lexicons, we should be able to model the finer grained aspects of children's preferences in the novel noun generalization task, for example, the greater robustness of the shape bias for solids than the material bias for nonsolids. However, there is also the possibility that when the network is given "messier" correlations, those correlations will not be strong enough to support the overarching solid-nonsolid distinction. Thus, it is an open question whether a network trained on a more realistic set of correlations will model children's performance in the novel noun generalization task. In Experiment 2, we attempted to model children's performance by using a more realistic training set than the idealized one used in Experiment 1 . We specifically considered three ways in which the correlations in Experiment 1 failed to match the correlations in the world.

First, the network in Experiment 1 was trained on an idealized version of children's vocabularies such that all lexical categories of solid things were organized by shape and all lexical categories of nonsolid things were organized my material. However, as reported by Samuelson and Smith (1999), the correlational structure among the nouns that English-speaking children learn early is not that clean. There are a considerable number of early nouns that do not follow these rules. For example, according to the adult judgments collected by Samuelson and Smith, bubble names a nonsolid shape-based category; soap names a solid material-based category; and nail, key, and crayon name categories for which both shape and material matter. Will a statistical learner be able to learn the general rules in spite of these exceptions?

Second, the network in Experiment 1 was trained on a corpus in which half of the lexical categories were for solid things and half were for nonsolid things. In contrast, children typically know many more names for solid objects than for nonsolid substances. This imbalance seems a likely factor in creating a shape bias that is more robust than the material bias. But are the few nonsolid material categories in this corpus enough for a simple neural network to learn to attend to material in the context of nonsolids, or will it overgeneralize attending to shape for both solids and nonsolids?

Third, when the training patterns for the network in Experiment 1 were constructed, additional correlations that are the natural product of differences between solids and nonsolids were not taken into account. In Experiment 1, the patterns representing shapes and materials for both solids and nonsolids did not differ in any systematic way. However, in the real world, solids can take a wide range of shapes, from simple to complex and from accidental pieces or flattened round shapes to ones with multiple parts, straight edges, and sharp angles. In contrast, nonsolids can take a much more restricted range of shapes, and these tend to be simpler 
than the shapes of solids. For example, wood can take the shape of logs, blocks, chairs, or ladders, but sand (as opposed to the container it is in) can take very few shapes. Being solid is thus less predictive of the particular shape an individual entity will take, and being nonsolid is more predictive. At the same time, shape is more predictive of category membership for a solid thing, but shape is less predictive of category membership for a nonsolid thing. Can a simple associative device find the correct higher level correlations given this added complexity? We addressed these issues in Experiment 2 by creating a training set that mimicked these properties: the imperfect correlations between solidity and category structure, the lopsided nature of children's early categories with many more names for solid things than for nonsolid things, and the correlation between nonsolidity and a restricted range of simpler shapes.

\section{Method}

All aspects of the architecture and the training and testing procedures in Experiment 2 were identical to those used in Experiment 1.

The modeled vocabulary. The goal of the training phase was to put in the network the vocabulary that a child brings into a novel noun generalization experiment. Accordingly, we first describe the structure of children's early vocabulary of object and substance terms, the structure we attempted to mimic in the network's training set. The training set was modeled on a set of 149 of the earliest learned object and substance names (Fenson et al., 1993). We characterized the category structure of each noun - its organization by shape or material-through the use of the judgments collected by Samuelson and Smith (1999). In addition, for each category we obtained judgments from three adults on the perceptual cues indicating solidity. We did this rather than use adults' characterizations of things as simply "solid" and "nonsolid" (as in Samuelson \& Smith, 1999) in order to model the correlations among the perceptual properties (and not the higher order notions of solidity-nonsolidity that might result from those correlations). Accordingly, adults were asked for each noun to answer the following three questions:
1. Do items named by the word change shape when pressed?

2. Do they return to their original shape after being pressed?

3. Do they take the shape of their container?

Each noun was then classified as referring to solid things if the three judges answered all three questions "no" or as referring to nonsolid things if all three judges answered the three questions "yes." Nouns with any other pattern of answers were categorized as perceptually ambiguous.

Figure 8 shows the relation between solidity-nonsolidity and category structure in this early learned vocabulary of object and substance terms. It is important that although adults judged most solid things to be categorized by shape, there were exceptions and complications; for example, muffins were judged to be alike in both shape and material, and bubbles were judged to be nonsolid but similar in shape. However, as is apparent, within this corpus of nouns and by these methods, solids tend to be named by shape, and nonsolids tend to be named by material.

The training set. The networks were trained on 24 categories that instantiated the same regularities (and irregularities) evident in our analysis of early nouns. Figure 9 shows the percentage of nouns of each kind (solid-shape, solid-material, nonsolid-shape, and nonsolid-material) for the training set for the network. As one can see by comparing Figure 8 and Figure 9, the statistical regularities present in the larger corpus were present in the smaller training set.

More specifically, the statistical regularities across the noun vocabularies were built into the network's training set in the following way. First, for each word that the network was to be taught, a pattern was generated to represent its value along the relevant dimension-the dimension on which objects named by that noun were judged to be similar. Second, at each presentation of the word, the value along the irrelevant dimension was varied randomly. For example, the word ball was judged to refer to things that are similar in shape; thus, a particular pattern of activation was randomly chosen and then assigned to represent ball-shape. All balls presented to the network were defined as having this shape, although each ball presented to the network also consisted of a unique and randomly generated pattern defining the material. So, each time the unit representing

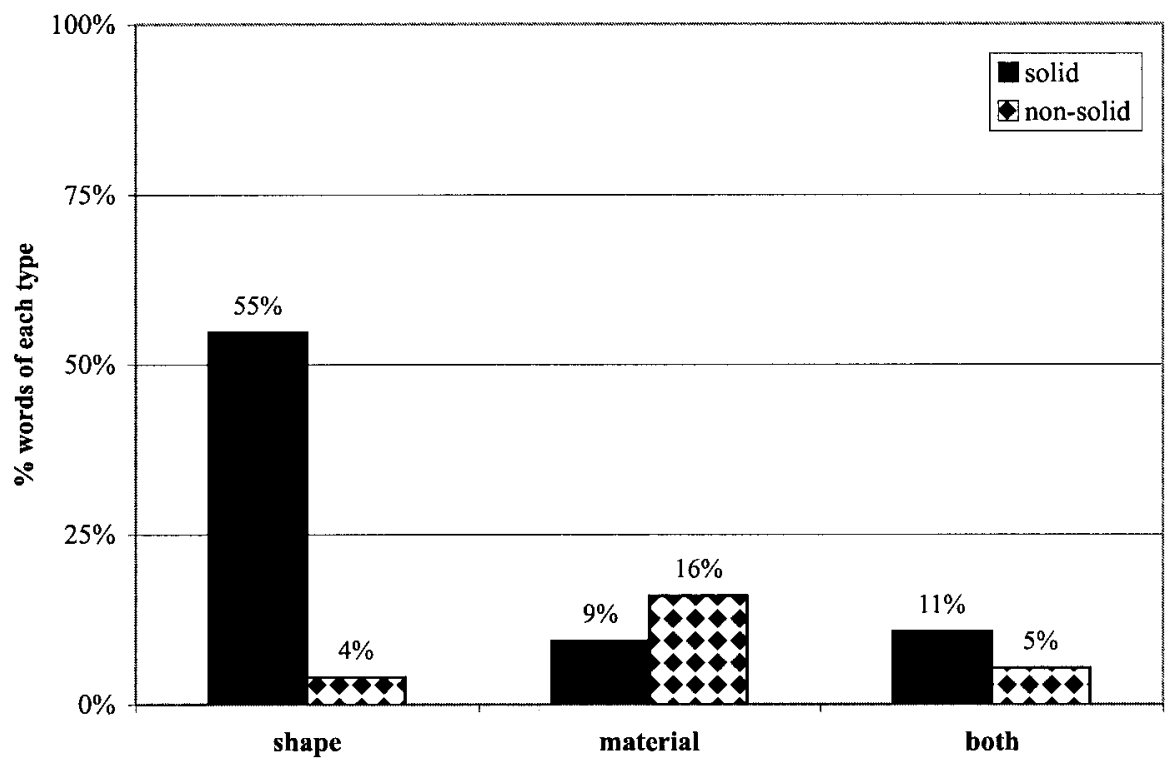

Figure 8. Percentages of early English nouns naming solid and nonsolid things judged by adults to refer to things alike in shape, material, or both. 


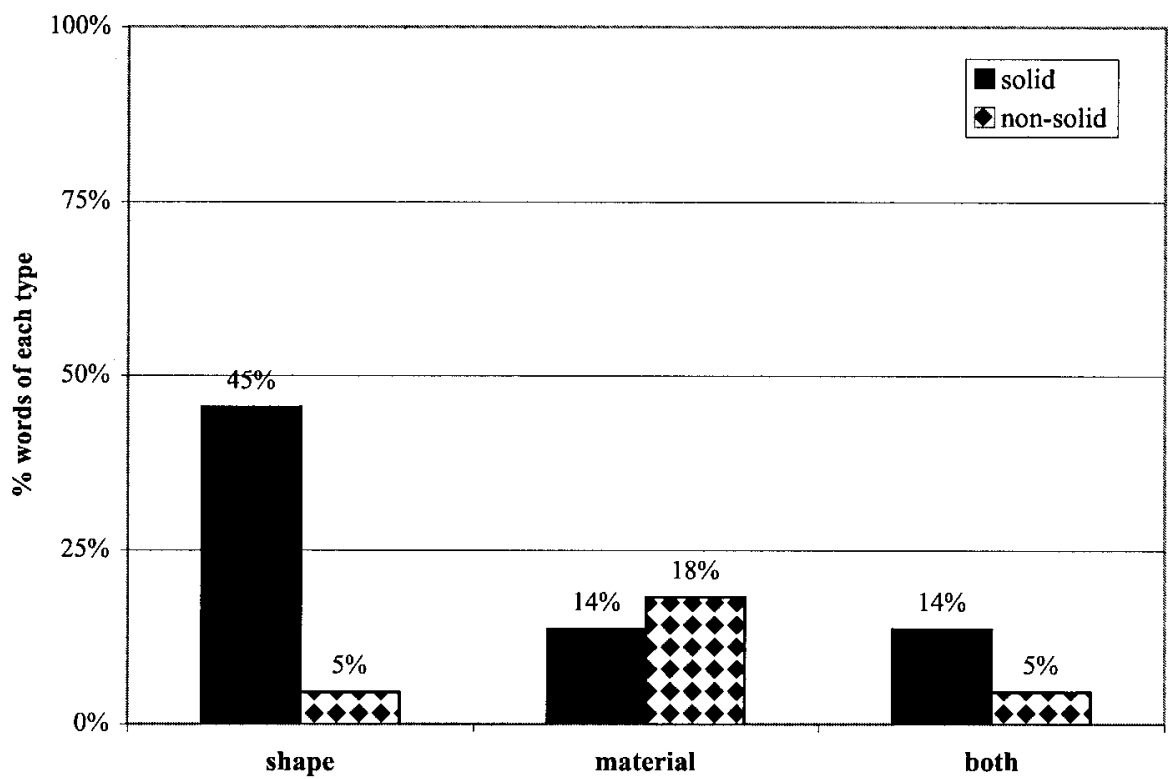

Figure 9. Percentages of training categories of solid and nonsolid things that were shape based, material based, or both.

the word ball was turned on during training, the pattern representing ball-shape was presented along the shape dimension, and a different randomly generated pattern was presented along the material dimension. Thus, all instances of ball shared the same shape pattern but had different material patterns.

To implement the fact that solid things can hold more varied and complex shapes than can nonsolid things, we assumed solid objects to have a bigger range of values along the shape dimension than nonsolid substances. Thus, instances of different solid lexical categories were very different in shape, but instances of different nonsolid lexical categories were not.

\section{Results}

The networks readily learned the words in the training vocabulary. Figure 10 shows the networks' performance on the training set as training progressed. The $y$-axis shows the percentage of

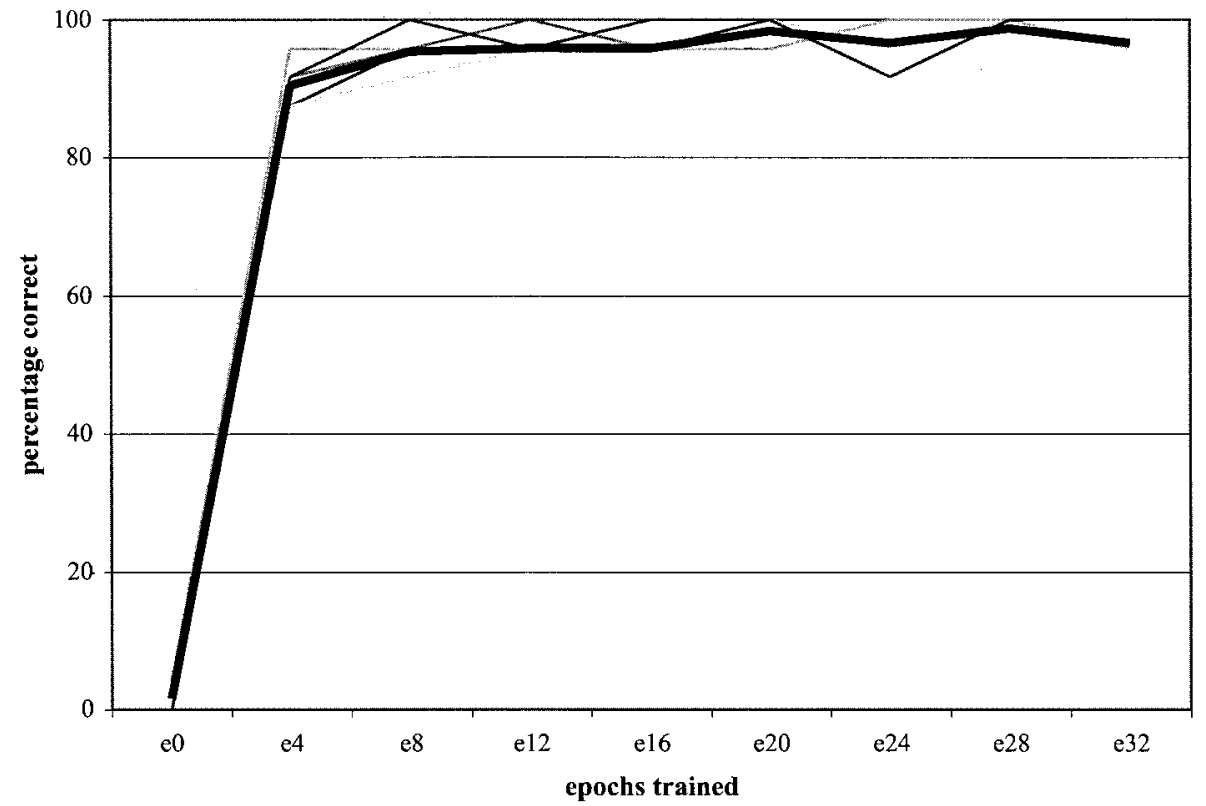

Figure 10. Correct productions of the target word given a training instance during the training phase for the 10 networks in Experiment 2. The thick line represents the average performance of the 10 networks. $\mathrm{e}=$ epoch. 
correctly labeled category instances; the $x$-axis shows number of passes through the training set. The networks approached ceiling performance after eight passes through the training vocabulary.

Figure 11 shows the proportion of shape choices in our simulated version of the novel noun generalization task before training and after being trained for 32 epochs. A paired $t$ test comparing the proportion of shape choices predicted by the networks on solid trials versus nonsolid trials revealed that, after training, the difference was significant, $t(9)=19.720, p<.0001$. More specifically, the trained connectionist networks showed a preference for shape matches for solids and a preference for material matches for nonsolids. The proportion of shape choices predicted for the solid trials was significantly greater than chance, $t(9)=8.315, p<$ .0001 , and for the nonsolid trials was significantly less than chance, $t(9)=-23.595, p<.0001$. That is, the networks were more likely to choose the shape match for a novel solid exemplar but were more likely to choose the material match for a novel nonsolid exemplar. All of the 10 networks showed this pattern, developing a shape bias for solids and a material bias for nonsolids.

We also analyzed the proportion of shape choices predicted by the networks for the patterns of the testing set prior to training. A paired $t$ test revealed a significant difference between the proportion of shape choices predicted for the solid trials and the shape choices predicted for the nonsolid trials, $t(9)=14.965, p<.0001$. Comparing the networks' predictions to chance revealed chance performance on the solid trials, $t(9)=0.168, p=.86$, but a proportion of shape choices significantly less than predicted by chance for nonsolid trials, $t(9)=-14.419, p<.0001$. In other words, the shape bias was not there at the start for the networks, but it emerged as a consequence of learning. However, in contrast with the results in Experiment 1, these networks showed a material bias for nonsolids before the stimuli had been trained at all. This result contrasts sharply with the developmental evidence that indicates that for children, the material bias emerges later than the shape bias (Samuelson \& Smith, 1999; Subrahmanyam et al., 1999).

Why might a material bias for nonsolids preexist word learning in the present simulations but not in the experimental tests of children's biases? The reason for this early material bias in the networks is evident when one looks at the networks' internal representations. Figure 12 shows the average distance between IR vectors for patterns representing solid things with the same shape, solid things of the same material, nonsolid things of the same shape, and nonsolid things of the same material before and after training. For solids, prior to training, instances that match in shape are not more similar to each other than are those that match in material. It is training that causes the shape of solid inputs to be emphasized so that the network's representations of two patterns for solids that have the same shape but different material are more similar to each other than its representation of two patterns for solids of the same material but different shape. However, for nonsolids, before training, the distance between IR vectors for patterns that represent the same material but different shape is much smaller than the distance between IR vectors representing the same shape but different material. The cause is straightforward. This greater similarity for nonsolids matching in material than for nonsolids matching in shape results from the restricted range of shapes that nonsolids can take.

This assumption was built into the training set because it seemed to characterize a real difference between solid and nonsolid shapes in the world and because it was unclear a priori whether this added complexity would help or hinder the formation of the two biases. It is critical that if this characterization of the perceptual differences between the ranges of shapes of solid and nonsolid things is correct, then the simulations predict that children-like the net-

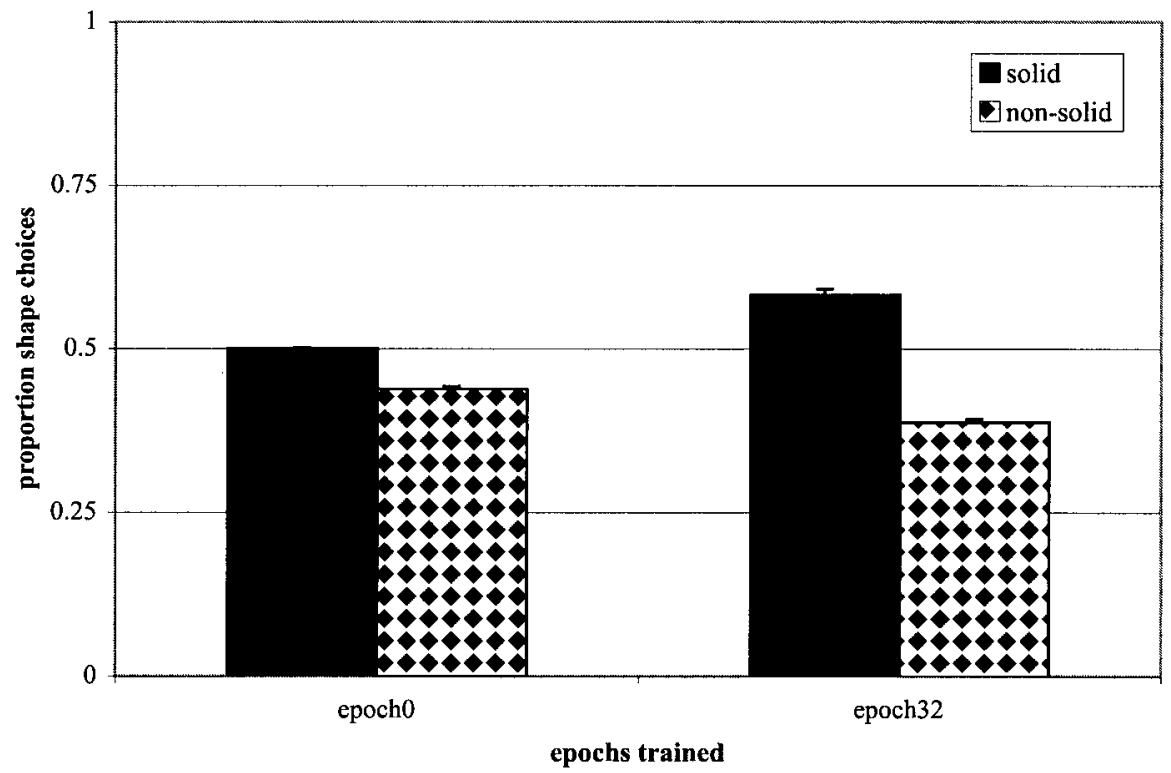

Figure 11. Mean proportion choices of the shape-matching test item by the networks for solid and nonsolid patterns prior to training (Epoch 0) and after training (Epoch 32) in Experiment 2. Error bars represent standard error. 


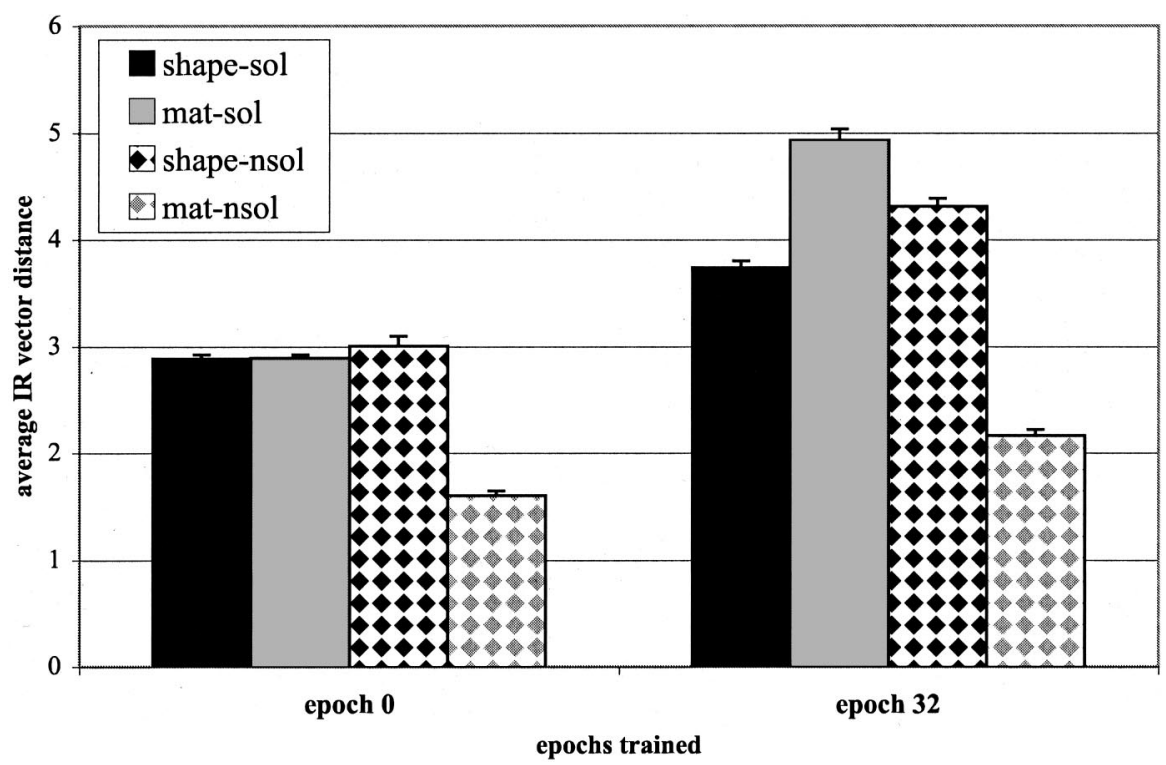

Figure 12. Raw similarity measures for the internal representations (IRs) for the four kinds of test items in Experiment 2. Error bars represent standard error. sol = solid; mat = material; nsol = nonsolid.

works-should have to learn a shape bias for solids but should appear to generalize names for nonsolids by material from the start if these materials are presented in the simpler and less constructed shapes typical of nonsolids. This seems a strange prediction because the existing evidence indicates a developmentally earlier shape bias than a material bias (e.g., Samuelson, 2002; Samuelson \& Smith, 1999; Subrahmanyam et al., 1999). One potentially relevant factor is the nature of the shapes of the nonsolids in the simulations and in the experiments. The shapes of nonsolids in the simulations were relatively similar to each other on the assumption that nonsolids naturally take accidental shapes that do not vary greatly. Experiments with children have typically involved somewhat more constructed shapes. In one experiment, Soja et al. (1991) varied the constructedness of nonsolid shapes and found no effect, but still their test sets were arranged such that the "unconstructed" shapes differed substantially from each other. At any rate, the results of the simulations suggest the issue merits reexamination.

If the predictions from the simulations are taken seriously, then they suggest the following: First, when very young children (who do not yet know many names for solid and nonsolid things) are given novel nonsolid things in the unconstructed shapes typical of nonsolids (splatters and smears), they will generalize names by material. This is predicted not because these children know anything about the importance of material for nonsolids but simply because the shape differences for these unconstructed solids are relatively small and thus the relative overall similarity between any two nonsolids in accidental shapes will be determined mostly by material similarity. Second, when given nonsolid things in constructed shapes, very young children (who do not yet know many names for solid and nonsolid things) will not attend to material because they have not yet developed a generalized expectation to name nonsolid things by material. This generalized expectation, like the generalized shape bias, must be learned from learning names for things. We tested these predictions in Experiment 3.

\section{Experiment 3}

Children younger than 30 months (between the ages of 18 and 28 months) were selected for this experiment because the goal was to test the predictions of the networks prior to mastery of the vocabulary used as the basis for the training sets for the networks, a vocabulary that by normative standards is known by $50 \%$ of 30-month-olds (Fenson et al., 1993). In this experiment, the children were presented with novel nonsolid materials either in the typical roundish, irregular shapes of nonsolids or in the constructed, sharp-edged shapes more typical of solids.

\section{Method}

Participants. Twelve children between the ages of 18 and 28 months were recruited.

Stimuli and design. The shapes and materials used in this experiment are shown in Figure 13. The natural shapes were a round mound and a thick smear; the constructed shapes were a squared $U$ shape and a circle with a slice cut out. The materials for one stimulus set were (a) Noxzema mixed with coarse brown sand and (b) shaving cream mixed with fine colored sand. The materials for the second set were (a) toothpaste mixed with glitter and (b) frosting. All children saw both a natural set and a constructed set. To control for the saliency of different materials, we assigned half of the children at random to judge the natural version contrasting Noxzema and shaving cream and the constructed version contrasting toothpaste and frosting. The remaining children had the opposite assignment. The two exemplars were presented in separate blocks. Each shape-match/materialmatch pair was presented four times, for a total of eight trials. The materials and order of sets were counterbalanced across participants; the position of the choices was counterbalanced across trials.

Procedure. The procedure used was a forced-choice task. The children were shown an exemplar (e.g., the Teema) and told its name ("This is the Teema"). The children were then presented with pairs of objects, a shape match and a material match, and were asked, "Can you show me the Teema?" Children were allowed to touch the stimuli if they showed interest in doing so. If the shapes were distorted, the experimenter reformed them 


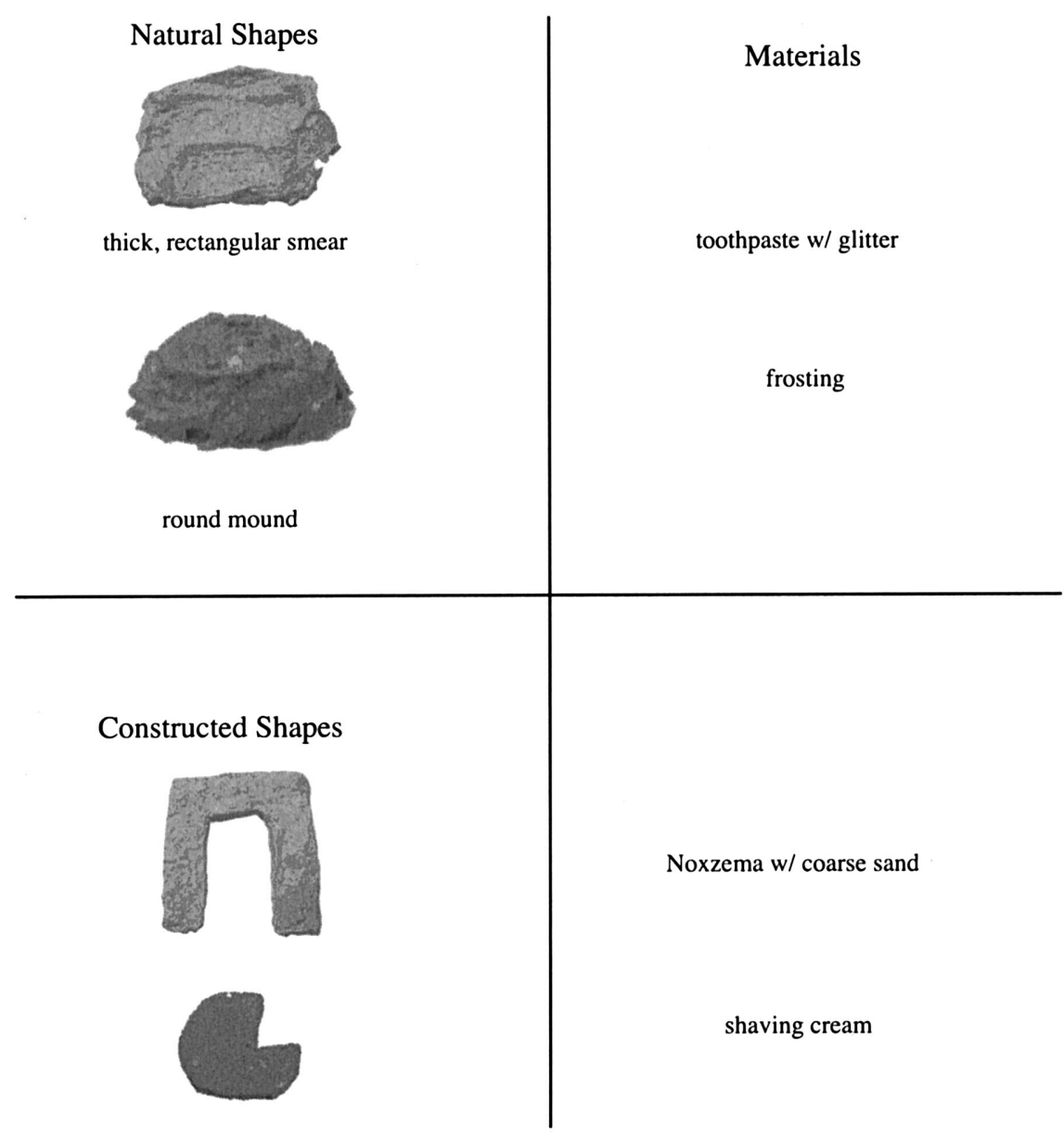

Figure 13. Stimuli for Experiment 3.

out of the children's view before continuing with the experiment. Children's responses were coded as the first object they touched or to which they pointed.

\section{Results}

Figure 14 shows the proportion of material choices for the natural and the constructed sets. Children were more likely to choose the material match when the nonsolid materials were presented in the shapes typical of nonsolids than when the nonsolid materials were shaped into more angular and solid-looking shapes, $t(11)=3.855, p<.01$. Also, with the natural set, children were more likely to choose the material match than would be expected by chance, $t(11)=3.071, p<.01$. However, in the constructed set, children's choices did not differ from chance, $t(11)=-1.483, p>$ .1. This experiment shows that if the shapes used are the shapes typical of nonsolids, then young learners do form categories of nonsolids by material. However, this is not a generalized bias that extends to nonsolids in more constructed shapes. These results suggest that the assumption about the different ranges of possible shapes for solid and nonsolid materials may be right. That is, with unconstructed nonsolids, the children show an early and perhaps unlearned preference for material matches-just as the untrained network does.

\section{Summary of Experiments 1-3}

The simulations in Experiments 1 and 2 demonstrated the mechanistic plausibility of the associative learning account, and the results from the children in Experiment 3 provide support for our assumptions about the shape regularities that characterize solids and nonsolids. In Experiments 1 and 2, we showed that networks trained on a biased vocabulary become biased. From learning specific instances of categories of solid things similar in shape and specific instances of categories of nonsolid things similar in material, the network learned to attend to the shapes of even novel solid things and to the materials of even novel nonsolid things. Moreover, Experiment 2 showed that the noisy regularities that characterize the early English noun corpus, imperfect as they are, may be sufficient to create these more abstract generalizations Finally, these experiments also highlight the value of simulations and the value of trying to take seriously the regularities in the early noun corpus. The simulations in Experiment 2 with the assumptions about the nature of the input led to a new prediction about an early unlearned material bias for nonsolids in unconstructed shapes. The findings not only support the prediction but also point to the importance of shape-constructed or unconstructed-in children's category formation, a factor that some have taken to be 


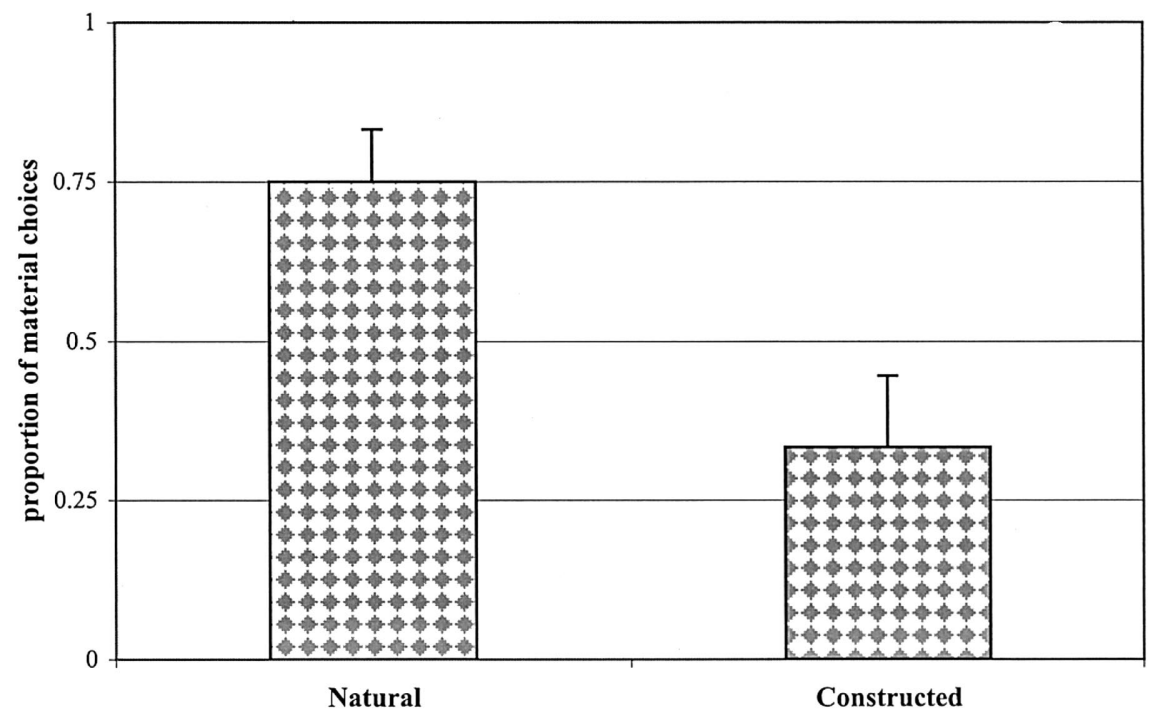

Figure 14. Mean proportion of material choices by the children given nonsolid exemplars in natural and constructed shapes. Error bars represent standard error.

essential to mature concepts of object and substance (Gelman \& Bloom, 2000; Prasada et al., 2002).

\section{A New Prediction: The "Ontology" Bias \\ Experiment 4}

One pervasive regularity in the early noun lexicon is that things that share the same name share the same solidity - for example, all tables are solid, all juice is nonsolid, and all wood is solid. Although any material can, in principle, be solid or nonsolid (depending on the temperature), names tend to not refer to categories that span the solid-nonsolid boundary. Thus, liquid water is "water," but solid water is "ice." This tendency is very strong in early child vocabulary. In fact, it is true for all of the nouns in the early noun corpus except for one-egg, which adults judged to have both solid and nonsolid forms. If the networks and children are learning these statistical regularities, then they should adhere to this constraint in their novel noun generalizations. That is, after training, the networks should act as if solids and nonsolids are different kinds that cannot be in the same category. We call the hypothesized bias to restrict categories to items of the same solidity the ontology bias because, like a hypothesized psychological ontology, such a bias would operate to divide entities into nonoverlapping kinds.

The rationale for our method to test this hypothesized bias is clarified by thinking about the typical way the shape bias for solids and the material bias for nonsolids are measured in the novel noun generalization task. Figure 15a shows an example of a typical task trial with solids. The child is shown a wooden $U$ shape and told its name. The child is then asked to choose which of a wooden $L$ shape or a plastic $U$ shape would also be called by the same name. Thus, the child has to choose whether the name refers to the shape (U shape) or the material (wood) of the named object. Notice that in this case, the shape match is a match in both solidity and shape, and the material match is a match in both solidity and material. In the absence of a solidity mismatch, the evidence indicates that children choose the shape match for a solid object. The question is, Will children still choose the shape match if it no longer matches a)
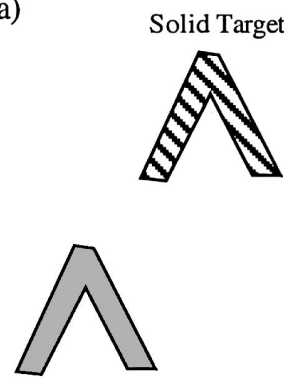

shape match solidity match

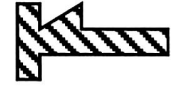

material match solidity match b)
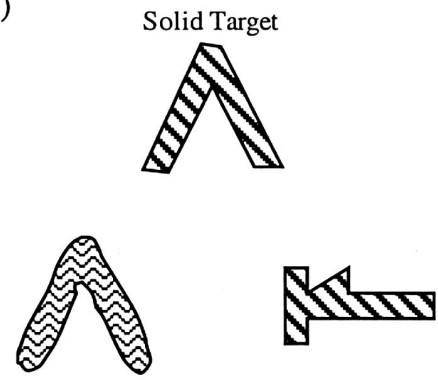

$\begin{array}{ll}\text { shape match } & \text { material match } \\ \text { solidity mismatch } & \begin{array}{l}\text { solidity match }\end{array}\end{array}$

Figure 15. Structure of the (a) same-solidity trials and (b) cross-solidity trials for solid targets. 
in solidity, if it violates the ontology bias? Figure $15 \mathrm{~b}$ shows an example of a cross-solidity set. In contrast to the traditional samesolidity sets, in the cross-solidity set the shape match is constructed from a nonsolid material such as shaving cream, but the material match is still the same, constructed of wood. Thus, the shape match is no longer a solidity match, but the material match is still both a material and a solidity match. In this experiment, we tested this prediction with networks, and in the next two experiments with children. If the networks (and the children) have learned the regularities in the lexicon involving solidity, and if learning these regularities creates overhypotheses or second-order generalizations, then networks (and children) should be constrained to extend names to other things of the same solidity.

\section{Method}

Architecture and training. The network architecture, training set, and training procedure were the same as those used in Experiment 2.

Testing. The testing procedure closely followed the procedure in Experiment 2 . However, the specifics of the test patterns were changed to make the cross-solidity test, as shown in Figure 16. The same-solidity trials were the same as in Experiment 2: Both the novel exemplar and the two test objects shared the same solidity (all three were solid or all three were nonsolid). In contrast, for the cross-solidity trials, the novel shape test match for solid exemplars was defined as nonsolid, and the novel material test match for nonsolid exemplars was defined as solid. Defining a pattern as solid or nonsolid simply meant activating the corresponding unit in the solidity layer of the network. Thus, the only difference between the same-solidity test patterns and the cross-solidity test patterns was the activation pattern along the solidity layer for the shape match in the case of solid trials and for the material match in the case of nonsolid trials-a difference of one bit. So, on the cross-solidity solid trials, we compared the network's preferences for a solid material match versus a nonsolid shape match, and on the cross-solidity nonsolid trials, we compared the network's preferences for a nonsolid shape match versus a solid material match.

As in Experiment 2, on each test trial, a novel exemplar was created by randomly generating an activation pattern along the shape and material dimensions. Shape and material matches were created by combining the exemplar's shape and material patterns with randomly generated material and shape patterns, respectively. Again, the networks were tested on 40 novel exemplars, half of them defined as solid and half of them defined as nonsolid.

\section{Results}

Figure 17 shows the proportion of shape choices predicted by the networks for solid exemplar trials and for nonsolid exemplar trials. These proportions for each of the 10 networks in each condition were submitted to a 2 (solidity: solid vs. nonsolid exemplar) $\times 2$ (set: same solidity vs. cross-solidity) mixed design. The analysis revealed a main effect of solidity, $F(1,18)=148.12$, $p<.001$; a main effect of set, $F(1,18)=13.98, p<.001$; and a reliable Solidity $\times$ Set interaction, $F(1,18)=58.13, p<.001$. In addition, the networks' predictions were compared against chance. In the same-solidity set condition, the networks predicted more shape choices than expected by chance on the solid trials, $t(8)=$ $12.551, p<.001$, and more material choices than expected by chance on the nonsolid trials, $t(8)=-12.872, p<.001$. In contrast, on the cross-solidity trials, the networks predicted chance performance, $t(8)=1.396, p=.2$, for solids; $t(8)=1.717, p=.1$, for nonsolids. That is, when the exemplar was solid, the networks no longer preferred the shape match, and when the exemplar was nonsolid, the networks no longer preferred the material match. None of the individual networks showed a shape bias on the cross-solidity trials given a solid exemplar, and none of the individual networks showed a material bias on the cross-solidity trials given a nonsolid exemplar. Thus, the pattern of generalization observed in Experiment 2 (and typical in experimental tests of children) is now disrupted: The networks did not show a preference for shape on solid trials and did not show a preference for material on nonsolid trials. These trials break up the correlations between within-category sameness in solidity and shape and within-category sameness in nonsolidity and material, and in so doing disrupt the shape bias and the material bias.

Solid Target

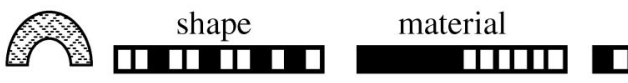

a) Same-solidity trials - Solid

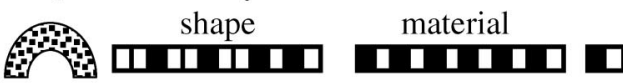

ט

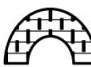

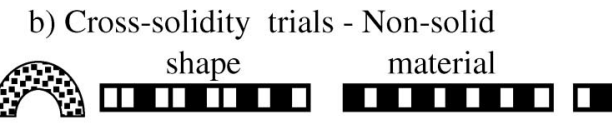

Amand 1 mmax

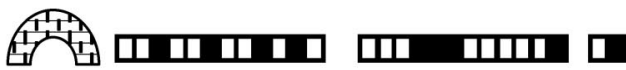

Figure 16. Representation of same-solidity (a) and cross-solidity (b) trials in the network. The only difference between the same-solidity test patterns and the cross-solidity test patterns is the activation pattern along the solidity layer for the shape match in the case of solid trials and for the material match in the case of nonsolid trials. 


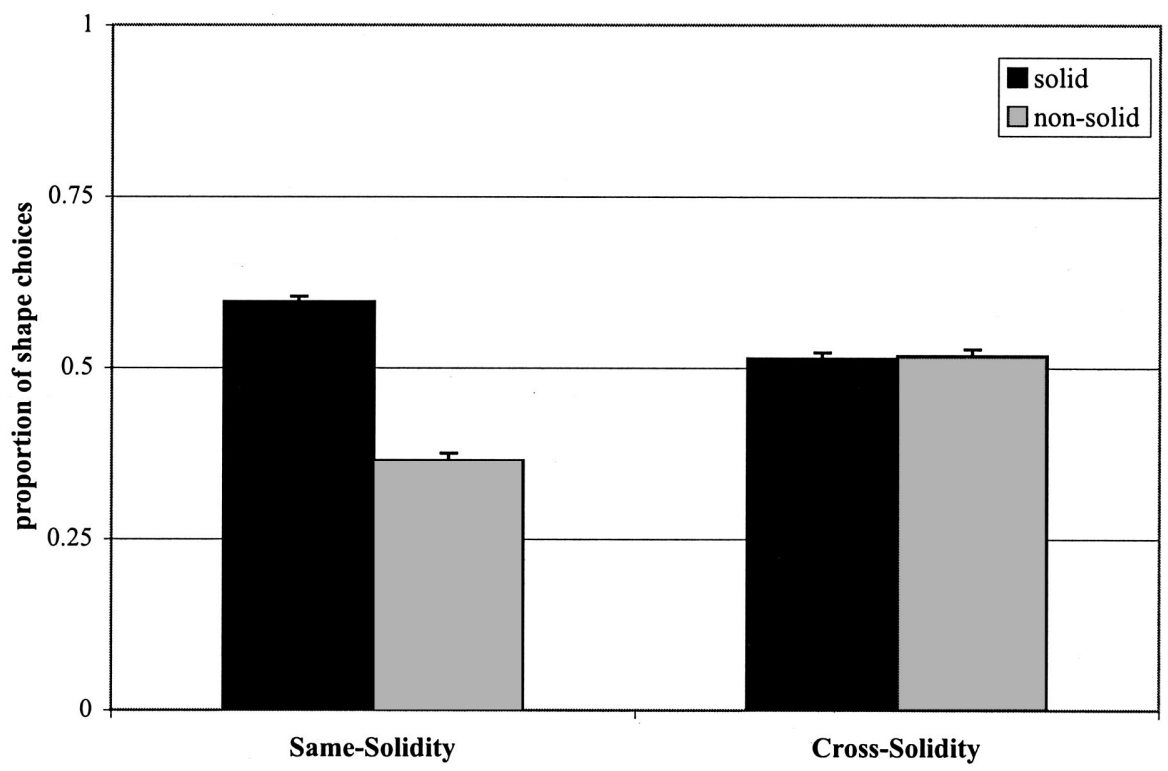

Figure 17. Proportion of shape choices predicted by the networks for the same-solidity and cross-solidity trials given solid and nonsolid exemplars. Error bars represent standard error.

\section{Experiment 5}

The question for this experiment was, Given a solid object, will children refuse to generalize its name to an object of the same shape if the test object is not solid? To test this question, we created solid exemplars with specific shapes and then tested objects of the same shape out of different solid materials or out of a nonsolid material. We contrasted these shape-matching test objects with ones that matched in solidity and material but differed in shape.

\section{Method}

Participants. Twelve children between the ages of 30 and 36 months participated. This age range was selected because the predictions derive from the performance of the networks after training on a vocabulary normatively known by $50 \%$ of children at 30 months.

Stimuli. The stimuli for Experiment 5 are shown in Figure 18. There were two exemplar objects. The exemplar for one set, the Teema, was a $U$ shape covered with red coarsely grained paint. The exemplar for the other set, the Wazzle, was an irregular M shape made of (rigid) blue cheesecloth. For each exemplar, there were three objects matching in material and two sets of items matching in shape. The same-solidity set consisted of three solid objects that matched the exemplar in shape and differed in material (e.g., metallic clay, Styrofoam covered with fur). The cross-solidity set consisted of shape matches made out of nonsolid materials (e.g., shaving cream, hair gel).

Procedure. The procedure used was a forced-choice task. The children were shown an exemplar (e.g., the Teema) and told its name ("This is the Teema"). The children were then presented with pairs of objects, a shape match and a material match, and were asked, "Can you show me the Teema?" Each child was presented with the same-solidity set of one exemplar and the cross-solidity set of the other. Half of the children were assigned at random to judge the same-solidity version of one exemplar and the cross-solidity version of the other. The two exemplars were presented in separate blocks. Each shape-match/material-match pair was presented twice in random order for a total of 12 trials. The order of the sets was counterbalanced across participants; the position of the choices was counterbalanced across trials.

\section{Results}

Figure 19 shows the proportion of shape choices for the crosssolidity and same-solidity sets. A $t$ test revealed a significant difference between the proportion of shape choices in the two sets, $t(11)=-2.22, p<.05$, indicating that the children attended to shape more in the same-solidity set than in the cross-solidity set. Comparisons to chance showed that on the traditional trials, the children selected the shape match at levels above chance that approached conventional levels of significance $(p<.10)$. Of the 10 children, 8 selected the shape match more than $75 \%$ of the time. On the cross-solidity sets-when the exemplar and material matches were solid but the shape match was nonsolid-the children were reliably more likely than chance to pick the solid material match $(p<.05)$. That is, they appeared to actively avoid the shape match if it meant putting a nonsolid thing in the same category as the labeled solid exemplar. These results are consistent with a bias in children to extend category names for solid things only to other solid things.

\section{Experiment 6}

Do children extend names for nonsolid things only to other nonsolid things and not to things of the same material that are solid? Figure 20 illustrates the three kinds of trials in Experiment 6. Given a nonsolid target, a shape match (also nonsolid) could be pitted against (a) a nonsolid material match (a same-solidity trial), (b) a solid material match in a simple shape (a cross-soliditynatural-shape trial), or (c) a solid material in a constructed shape more consistent with a solid thing (a cross-solidity-constructedshape trial). 


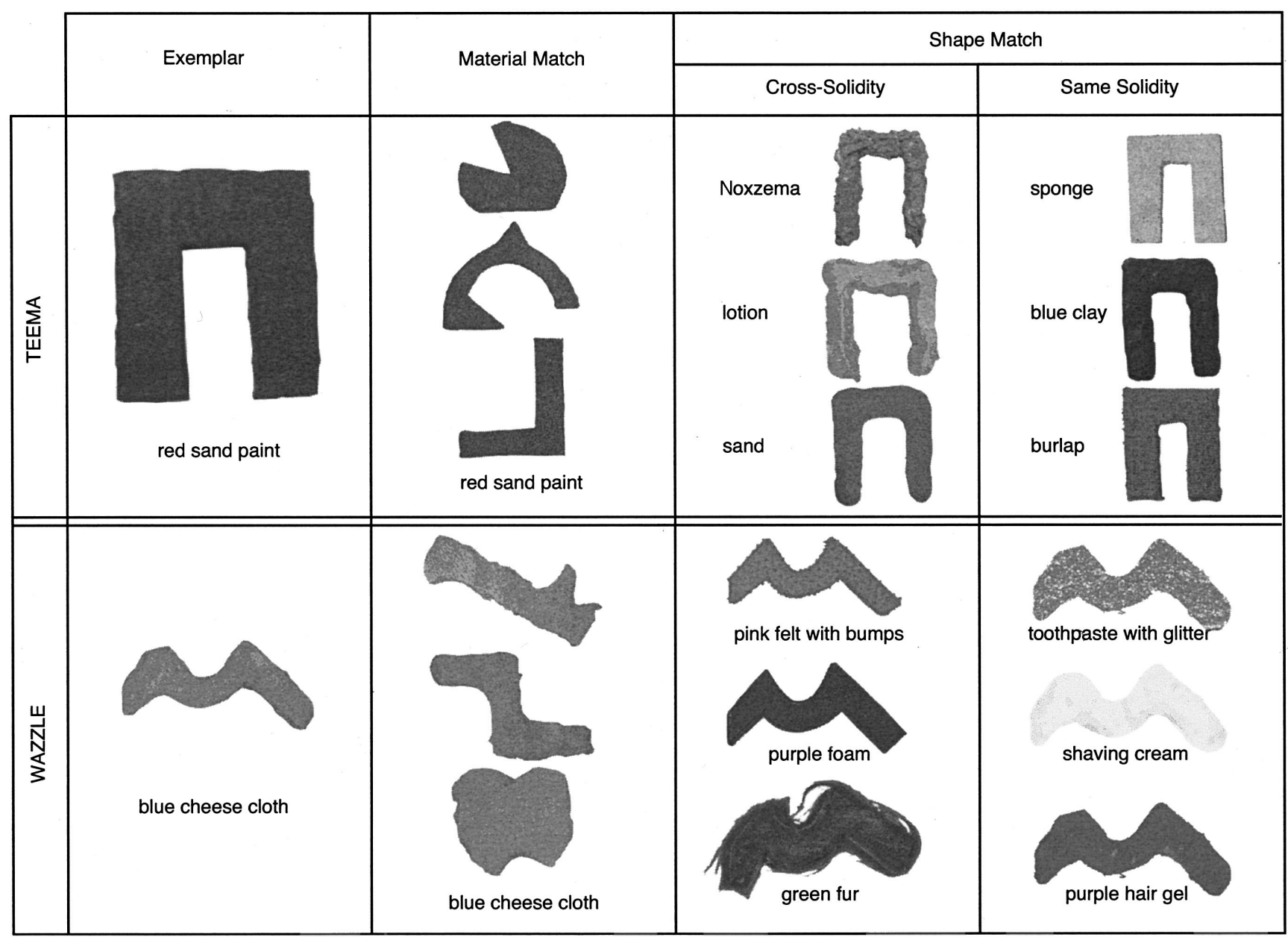

Figure 18. Stimuli for Experiment 5.

One key question is whether given a nonsolid exemplar, children will refuse to generalize its name to a material match if the test object is solid. A second question is the role of shape constructedness in these extensions. If children's expectations reflect the correlational structure in their vocabularies, then they should be more likely to choose the matching material when it is nonsolid (same-solidity condition) than when it is solid. Further, if they are sensitive to the correlation between kind of shape and category structure, then they should be more likely to choose the material match on the cross-solidity trials when the test object is solid but shaped like a nonsolid (cross-solidity-natural condition) than when it is solid and is shaped like a solid (cross-solidityconstructed condition).

\section{Method}

Participants. Eighteen children between the ages of 30 and 36 months participated in this study.

Stimuli. Many materials have solid and nonsolid forms that are not easily recognized by their perceptual properties as being the same material-for example, paper and paper pulp, copper and molten copper, or a muffin and muffin batter. Accordingly, judgments from six undergraduates were used to choose the solid and nonsolid versions of the "same material" that would appear to be the same material to observers. The resulting stimuli consisted of translucent gel and translucent hardened plastic for one set and off-white hand lotion and off-white hardened paint for the other. In both cases, the materials were judged by adults to be the nonsolid and hardened versions of the same material.

As shown in Figure 21, there were two exemplar objects. The exemplar for one set, the Teema, was a $V$ shape made out of translucent gel. The exemplar for the other set, the Wazzle, was an irregular $\mathrm{M}$ shape made out of hand lotion. For each exemplar, there was a set of shape matches made out of three different nonsolid substances. For the Teema, the shape matches were made out of wax, glitter, and Noxzema mixed with sand; for the Wazzle, the shape matches were made out of green sand, toothpaste with glitter, and shaving cream. For each exemplar, there were also three sets of material matches: a same-solidity set and two types of cross-solidity sets (natural shape and constructed shape). For the Teema, the samesolidity material match consisted of shapes made out of translucent hair gel, the cross-solidity-natural material match was a loose $\mathrm{S}$ shape made out of translucent hard plastic, and the cross-solidity-constructed material match was a square $S$ shape made out of the same translucent hard plastic. For the Wazzle, the same-solidity material match consisted of shapes made out of off-white hand lotion, the cross-solidity-natural material match was a kidney shape made out of off-white hardened paint, and the crosssolidity-constructed material match was an irregular T shape with straight edges and sharp angles made out of the same off-white hardened paint. 


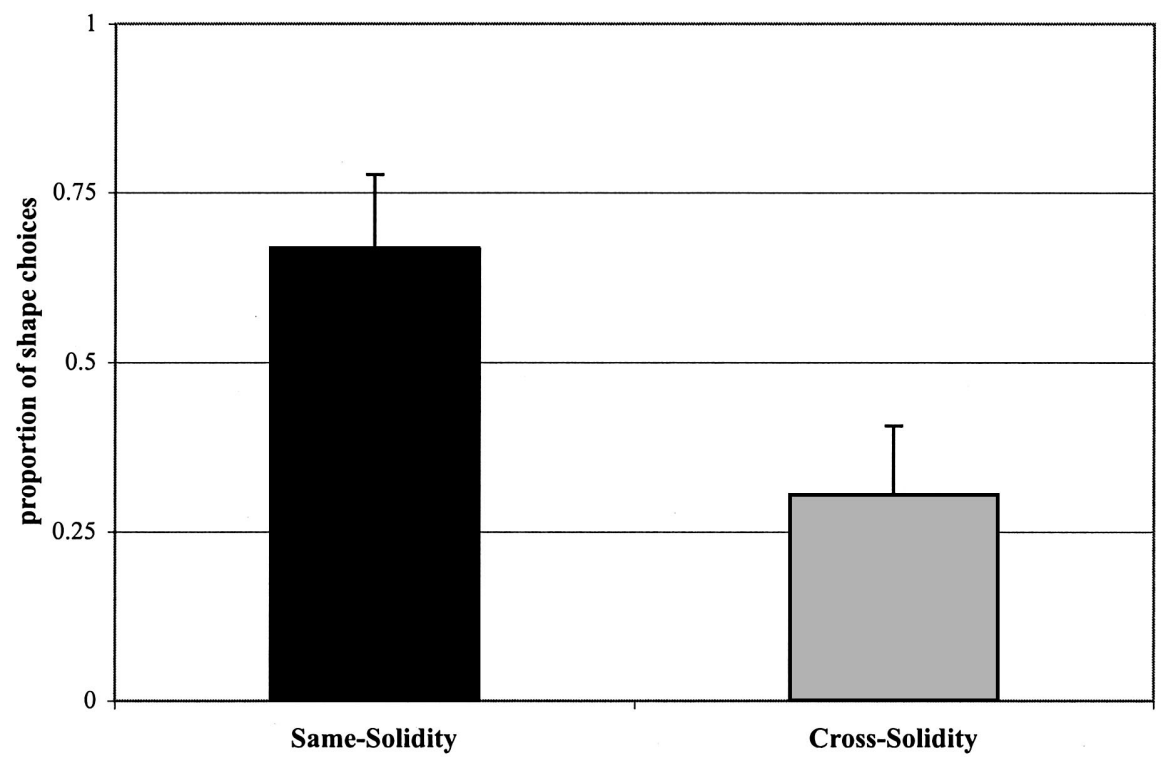

Figure 19. Mean proportion of shape choices for the same-solidity and cross-solidity sets in Experiment 5 (solid exemplars). Error bars represent standard error.

Procedure. The procedure was the same as that used in Experiment 5. Each child was presented with the same-solidity, cross-solidity-natural, and cross-solidity-constructed sets for the two exemplars. Each shapematch/material-match pair was presented twice in random order for a total of 12 trials. The children participated under one of two testing procedures. Six children saw the trials blocked by exemplar (e.g., they saw all three sets for the Teema and then all three sets for the Wazzle); the rest of the children saw the trials blocked by condition with the same-solidity, crosssolidity-natural, and cross-solidity-constructed sets counterbalanced. The order of the sets was counterbalanced across participants; the position of the choices was counterbalanced across trials.

\section{Results and Discussion}

Figure 22 shows the proportion of shape choices for the samesolidity, cross-solidity-natural, and cross-solidity-constructed sets. These proportions were submitted to a 2 (order: mixed or blocked) $\times 3$ (set: same-solidity, cross-solidity-natural, or crosssolidity-constructed) mixed design. The analysis revealed only a main effect of set, $F(2,34)=4.202, p<.05$. In the same-solidity trials, contrary to the networks' performances in Experiment 4, children performed at chance level, choosing the shape match and the material match equally often, $t(17)=-0.437, p>.5$. Although 2- to 3-year-olds in other experiments have shown a reliable material bias for nonsolids (Soja et al., 1991), there have been other reports of chance-level performance (Imai \& Gentner, 1997; Samuelson, 2002; Samuelson \& Smith, 1999; Subrahmanyam et al., 1999). The chance-level performance in the present experiment is perhaps not unexpected given the relatively constructed nature of the shapes of the named exemplars.

In the cross-solidity-natural trials, as the network simulations predicted, children also performed at chance level, $t(17)=1.236$, $p>$.2. That is, they did not extend the name of a nonsolid material to its solid version, even when the shape cues were consistent with nonsolidity. It is of interest that in the cross-solidity-constructed trials, children's performance was reversed-they chose the shape
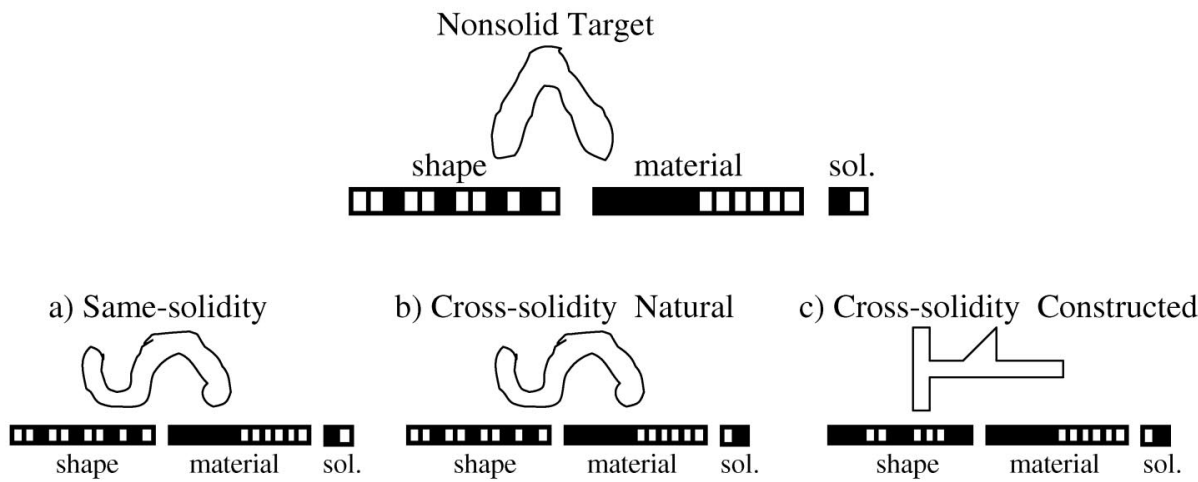

Figure 20. Structure of the (a) same-solidity trials, (b) cross-solidity-natural-shape trials, and (c) crosssolidity-constructed-shape trials. sol. = solidity. 


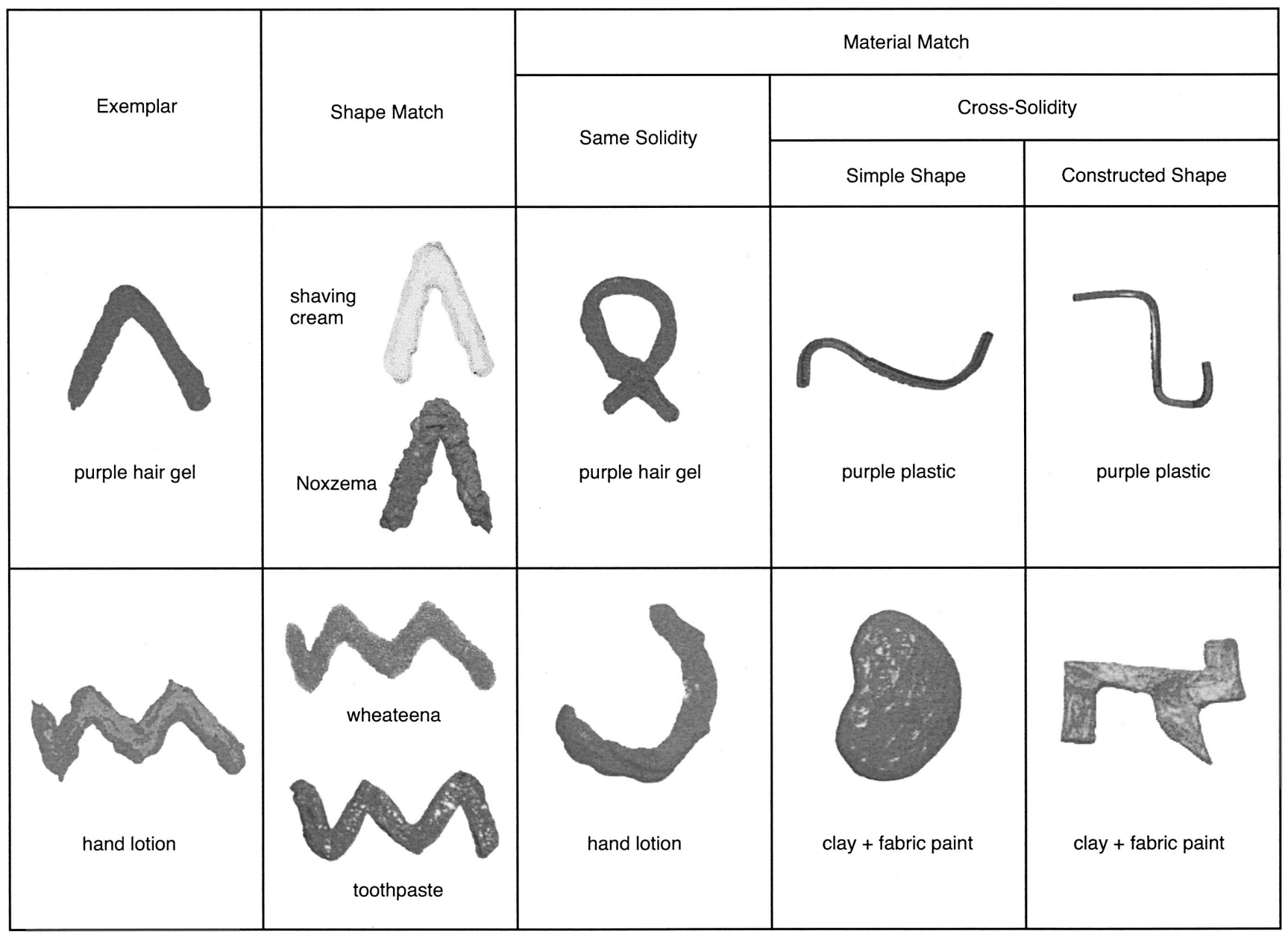

Figure 21. The stimulus sets for Experiment 6 (nonsolid exemplars). A shape-same-solidity match was pitted against three different kinds of material matches.

match more than expected by chance, $t(17)=2.287, p<.05$. That is, they grouped together two objects that matched in shape and were both nonsolid (but different materials) rather than grouping together two nonsolid things of the same material but different solidity and different constructed shapes. There were no significant effects of order, $F(2,34)=2.212, p>.1$. Notice, further, that in no case did children reliably form categories containing both solid and nonsolid things.

The difference between the cross-solidity-natural and the crosssolidity-constructed sets suggests that the kind of shape a nonsolid entity presents influences whether it is classified by shape or by material. In a follow-up simulation study, we confirmed that this was also true for the networks. In the previous simulation (Experiment 4 ), we altered only the solidity pattern; thus, the shape pattern remained one characteristic of nonsolid things. For these new simulations, we added a test set like the cross-solidityconstructed set; we switched the active unit in the solidity unit and used a shape pattern that was consistent with a solid thing as shown in Figure 22. These networks showed a material bias given the traditional sets (mean proportion material choices $=.60$, which differed from chance, $p<.05$ ), performed at chance level on the cross-solidity trials given the simply shaped solids (mean proportion shape choices $=.52$ ), but reliably chose the nonsolid shape match on the cross-solidity trials when the material match was complexly shaped (mean proportion $=.68, p<.05$ ). The networks, like the children, showed a preference for the same-solidity shape match over the cross-solidity material match in the crosssolidity-constructed trials but performed at chance level in the cross-solidity-natural trials-trials that pitted a choice object with a nonsolid material against one that was solid but had a shape consistent with nonsolidity. Thus, the networks, like the children, used the shape information as a cue to category structure.

\section{Summary of Experiments 4-6}

The early nouns that children learn contain multiple correlations among solidity and among kind of shape (simple or constructed), and moreover, these cues predict within-category similarities in solidity, shape, and material. The correlations available in the early noun corpus thus go beyond the generalizations that solid things are categorized by shape and nonsolid things are categorized by material. These three experiments demonstrated the existence of 


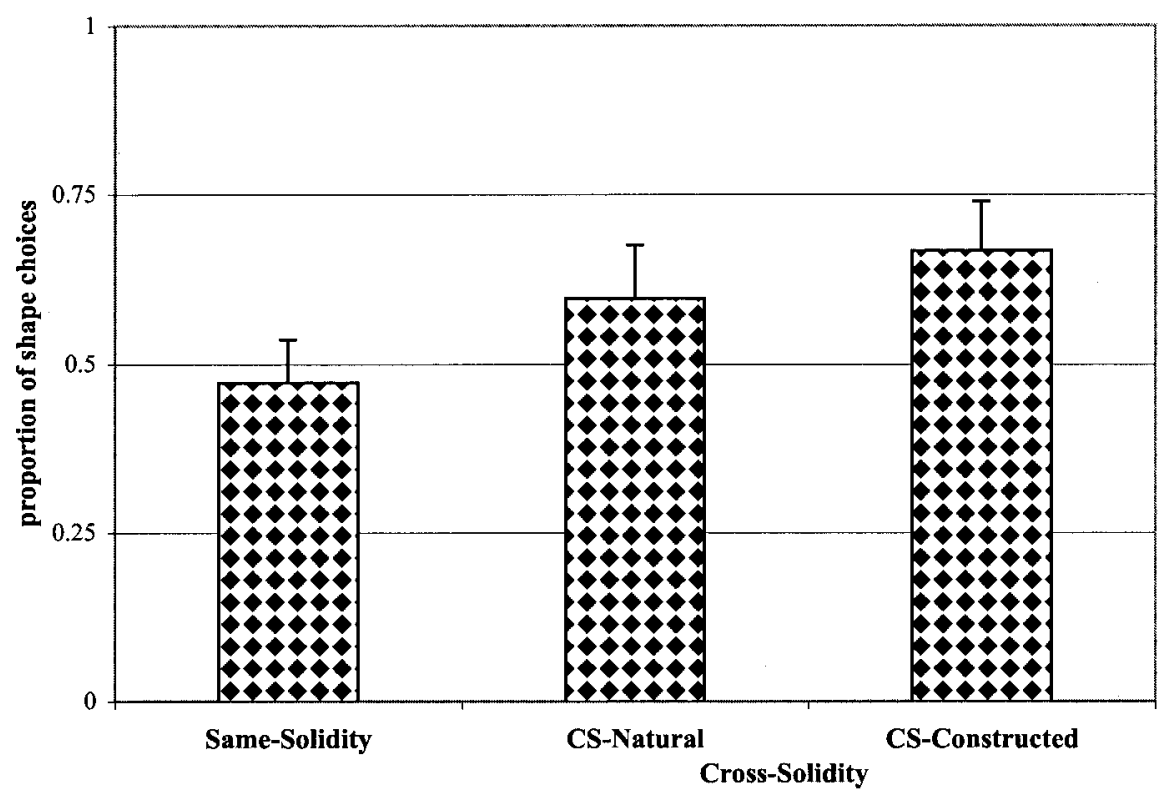

Figure 22. Mean proportion of shape choices by the children on the three different conditions of Experiment 6. Error bars represent standard error. CS = cross-solidity.

those correlations and their influence on children's novel noun generalizations. The results indicate that there is not simply a rule that shape matters for solids or that material matters for nonsolids. Instead, children's generalizations of a just-heard name for some novel thing is a product of all of these correlations. The strongest regularity in the present case is that solid and nonsolid things do not get the same name. Thus, children's novel noun generalizations are expected to show this bias even when other intercorrelated cues are put in conflict. We characterized this predicted tendency to put solids and nonsolids in different categories as an ontology bias, not because children's (or networks') judgments in this task reflect a represented ontology in the usual sense, but to underscore how higher order correlations in the early noun lexicon could create performances that seem to be generated by beliefs about fundamentally different kinds.

\section{Cross-Linguistic and Syntactic Issues}

If children's attentional biases in the novel noun generalization task are the product of correlations learned over the early lexicon, then these biases should be universal to the degree that the structure of early lexicons is similar across languages and should be different to the degree that the early lexicons differ. Past research (Imai \& Gentner, 1997; Kobayashi, 1997) has shown that children's novel noun generalizations exhibit both universal and language-specific properties. In the next three experiments, we ask whether we can explain both the universals and the differences with our associative learning account.

\section{Experiment 7}

Imai and Gentner (1997) showed that both English-speaking and Japanese-speaking children generalize names for solid and nonsolid things differently. We have shown that English-speaking children's performances in the novel noun generalization task are explainable by the correlational structure of the early noun lexicon. Can we also explain the biases shown in Japanese-speaking children by the correlations presented by the early Japanese lexicon? In this experiment, we begin answering this question by replicating Samuelson and Smith's (1999) analysis of the early English lexicon in Japanese.

\section{Method}

Participants. Sixteen adult native Japanese speakers participated in this study. Ten participants made category organization judgments, and the other 6 made judgments on solidity.

Stimuli. The nouns were selected from the Japanese Early Communicative Development Inventory, which is a parental checklist used to measure children's productive vocabulary. This checklist was developed from independent and extensive study of the common words and phrases known by children learning Japanese (Ogura \& Watamaki, 1997; Ogura, Yamashita, Murase, \& Dale, 1993). The words in this list are known by $50 \%$ of large samples of children at 30 months of age. Thus, the list is a good proxy for the kinds of words commonly known by young Japanesespeaking children. The object and substance terms on this list were specifically selected for this study. The nouns were selected following the same criteria as in Experiment 2. There were 167 nouns on the list.

Procedure. We followed Samuelson and Smith's (1999) procedure and asked adults to make judgments about the perceptual properties characteristic of the instances of each early noun category. For the solidity judgments, 6 participants were asked to answer, for each of the words in the vocabulary checklist, the following three questions:

1. Do items named by the word change shape when pressed?

2. Do they return to their original shape after being pressed?

3. Do they take the shape of their container? 
Each noun was then classified as referring to solid things if all three questions were answered "no" or as referring to nonsolid things if all three questions were answered "yes." Nouns with any other pattern of answers, which referred to things that were neither clearly solid nor clearly nonsolid (e.g., playdough and shirt), were categorized as ambiguous.

For the within-category similarity or category structure judgments, 10 participants judged each of the nouns in the list. For each noun, they answered the following questions:

1. Are the items in the category similar in shape?

2. Are they similar in color?

3. Are they similar in material?

Each noun was then classified as organized by shape if at least $70 \%$ of the participants agreed that the things named by that noun were judged to be similar in shape. A noun was classified as organized by material if participants agreed that the things named by that word were similar in material (and/or color). Note that each word could be classified as organized by shape, material, both, or neither. For comparison with English, we used the original data from Samuelson and Smith (1999) with the same criteria to select lexical categories of object and substance and the same criteria to categorize individual nouns as solid, nonsolid, or neither and as shape, material, both, or neither.

\section{Results}

The percentages of words of each kind-solid-shape, solidmaterial, nonsolid-shape, and nonsolid-material-are shown in Figure 23. In the following discussion, we compare the results of this study with the corresponding English data shown in Figure 9. As in English, the majority of the early Japanese nouns in this list refer to solid objects (78\% in Japanese, $75 \%$ in English), and there are very few nouns for nonsolids (21 in Japanese, 24 in English) in either list of early vocabulary items. In both languages, more nouns were judged to refer to things similar in shape (58\% in
Japanese, 59\% in English) than to things similar in material and/or color (15\% in Japanese, 25\% in English).

The correlations between solidity and category organization in the early Japanese vocabulary were also similar to those found in English. The correlation was very strong for solid and shape, with most of the words that were classified as solid also judged to refer to things that were similar in shape (96\% in Japanese, $88 \%$ in English) and most words that were classified as referring to things similar in shape also were classified as solid $(95 \%$ in Japanese, 93\% in English). The correlation was weaker for nonsolid and material. Whereas words that were classified as nonsolid were judged to refer to things that were similar in material $(90 \%$ in Japanese, $84 \%$ in English), the correlation did not hold in the opposite direction (46\% in Japanese, $51 \%$ in English). Thus, as in English, for the majority of early Japanese nouns, solidity and within-category similarity agreed in a way consistent with the shape and material biases, but not perfectly. The similarity of the two early noun corpora is remarkable in its own right, a fact we consider in the General Discussion. We know from Experiment 2 that these regularities are sufficient to create the shape and material biases in English-speaking children. Are they sufficient across the two languages to create both the similarities and the differences in English- and Japanese-speaking children's novel noun generalizations?

\section{Experiment 8}

Imai and Gentner (1997) found both similarities and differences in the way Japanese- or English-speaking children generalize novel nouns. We show their results for 2-year-old Japanese- and English-speaking children in Figure 24, the youngest children showing cross-linguistic differences in their study. We concentrated on these children with the idea that these cross-linguistic differences might be explainable solely by the correlations in the

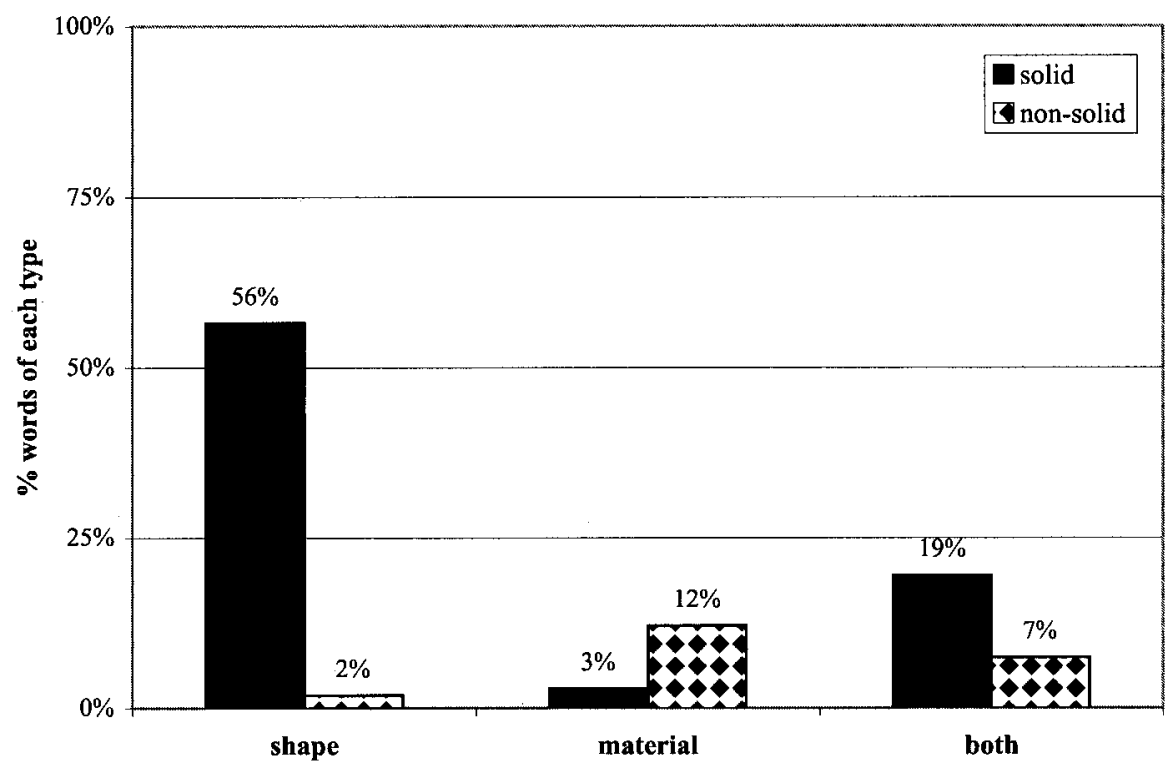

Figure 23. Percentages of early Japanese nouns naming solid and nonsolid things judged by Japanese-speaking adults to refer to things alike in shape, material, or both. 


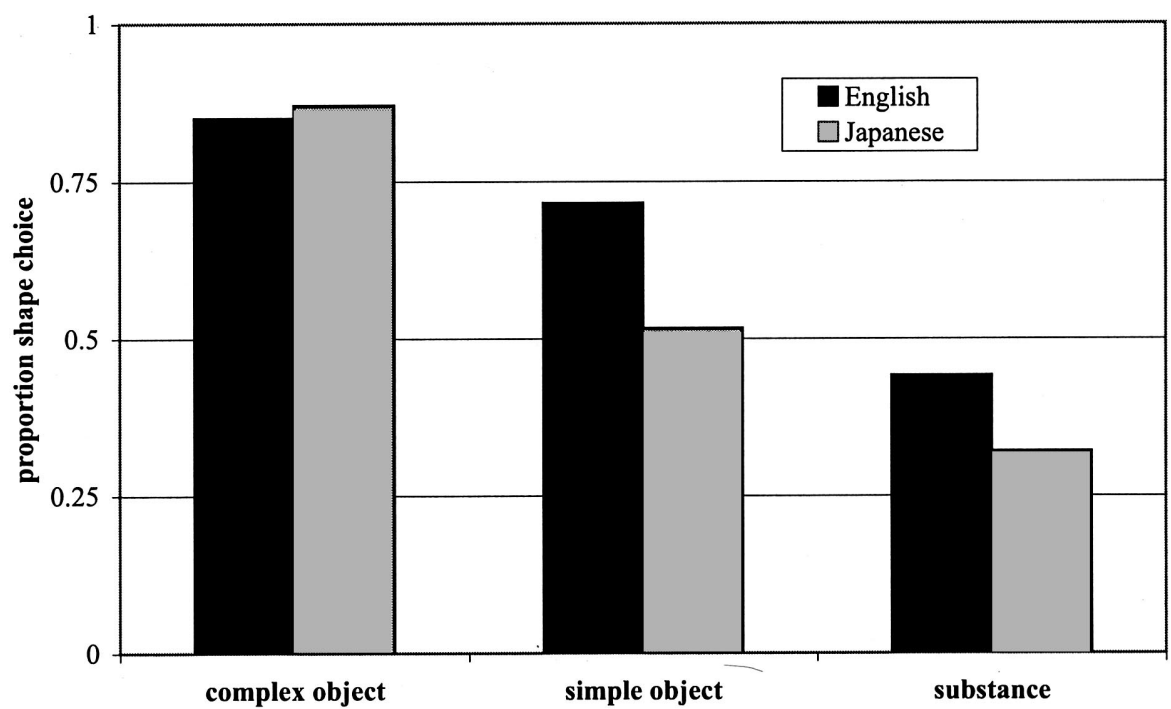

Figure 24. Two-year-old English- and Japanese-speaking children's shape choices in the three stimulus conditions from Imai and Gentner (1997).

noun lexicon. As Figure 24 shows, Japanese- and Englishspeaking children generalized names in the same way given clear cases of solid objects with constructed shapes or clear cases of nonsolid substances in natural shapes, but they generalized names for the simply shaped solids in different ways. For the Englishspeaking children, solid things-both complexly and simply shaped-were categorized by shape. For Japanese-speaking children, simply shaped things_-both solid and nonsolid-were more likely to be categorized by material. To determine whether these differences could be created by differences in the lexicons alone, we trained networks with vocabularies modeled after the English and the Japanese early nouns. The networks were tested on three kinds of test patterns: solid with shapes typical of solid things (complex shapes), nonsolids with shapes typical of nonsolids (simple shapes), and solids with shapes typical of nonsolids (simple shapes).

\section{Method}

Architecture and training. The network architecture, training set, and training procedure were the same as those used in Experiment 2 for the English training set (except the networks were additionally tested on solids with simple shapes). The same procedure used in Experiment 2 was used to turn the regularities in the early Japanese lexicon into the Japanese training set, as shown in Figure 25.

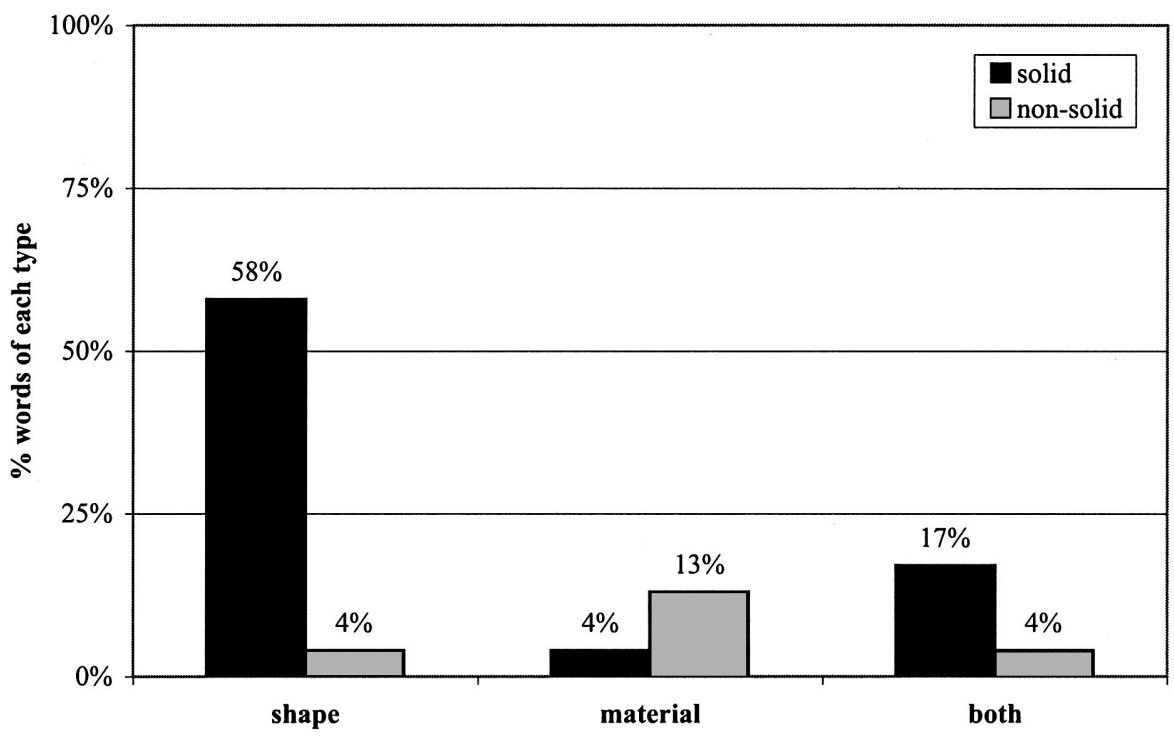

Figure 25. The Japanese training set: Percentages of training categories of solid and nonsolid things that were shape based, material based, or both. 
Testing. The testing procedure closely followed the procedure in Experiment 2. Test trials were of three kinds: exemplars defined by patterns of activation representing solid and complexly shaped things, solid and simply shaped things, and nonsolid and simply shaped things. Shape complexity was represented as in Experiment 2. The activation patterns along the shape dimension of simply shaped solids or nonsolids were drawn for a reduced space of possible shapes; they all had the shape that was defined as characteristic of nonsolid shapes. Complexly shaped test patterns were drawn from the unrestricted space. As in Experiment 2, on each test trial, we created a novel exemplar object by randomly generating an activation pattern along the shape and material dimensions and then created shape and material matches by combining the exemplar's shape and material patterns with randomly generated material and shape patterns.

\section{Results}

The performances of the networks are shown in Figure 26 for the Japanese training set and for the English training set. The light bars show children's proportion of shape choices; the dark bars show the probability of choosing the shape match predicted by the networks. These proportions of shape choices for each of the 10 networks for each language were submitted to a 3 (test set) within-subject analysis of variance. The analysis of the Japanese networks revealed a main effect of test set, $F(1,18)=542.316$, $p<.0001$. The networks were more likely to choose the shape match in the complex solid trials than in the simple solid or nonsolid trials. In addition, the networks' predictions were compared against chance. For the complex solid trials, the Japanese networks predicted more shape choices than expected by chance, $t(9)=25.237, p<.0001$; for the simple solid trials, their performance did not significantly differ from chance, $t(9)=-2.011$, $p=.07$, and for the nonsolid trials, the networks predicted less shape choices than expected by chance, $t(9)=-4.637, p=.001$. Eight of the 10 networks trained with categories modeled after early Japanese nouns showed this pattern; 2 of the networks developed shape biases for solid complex things but did not develop reliable shape or material biases for simple solids or nonsolids. Overall, then, the connectionist networks were successful in qualitatively modeling the pattern of performance of the 2-year-old Japanese-speaking children in Imai and Gentner's (1997) experiment.

The analysis of the English networks revealed similar results. There was a main effect of test set, $F(1,18)=223.707, p<.0001$. The networks were more likely to choose the shape match on the complex solid trials than on the simple solid or nonsolid trials. In addition, the networks' predictions were compared against chance. For the complex solid trials, the networks predicted more shape choices than expected by chance, $t(9)=8.315, p<.0001$; for the simple solid trials and for the nonsolid trials, the networks predicted less shape choices than expected by chance, $t(9)=$ $-11.601, p<.0001$, for simple solids and $t(9)=-10.894, p<$ .0001 , for nonsolids. Unlike the English-speaking children, the networks trained on the English-like vocabulary treated simple solids like nonsolids and unlike complex solids. All of the 10 networks showed this pattern. Thus, the English-trained networks yielded performances that fit the Japanese-speaking children's performances but not those of English-speaking children.

To explain the preferences of English-speaking children, we need something in the English training that puts both complexly shaped and simply shaped solid objects together and differentiates them from nonsolids. The obvious place to look is count-mass syntax.

\section{Experiment 9}

Can the regularities of English count-mass syntax alter the networks' development of learning biases and thus account for children's behavior in the case of simple solid objects? To pursue this issue, we trained networks with the English-like vocabulary used in the previous English simulations adding the correlated syntax cues.

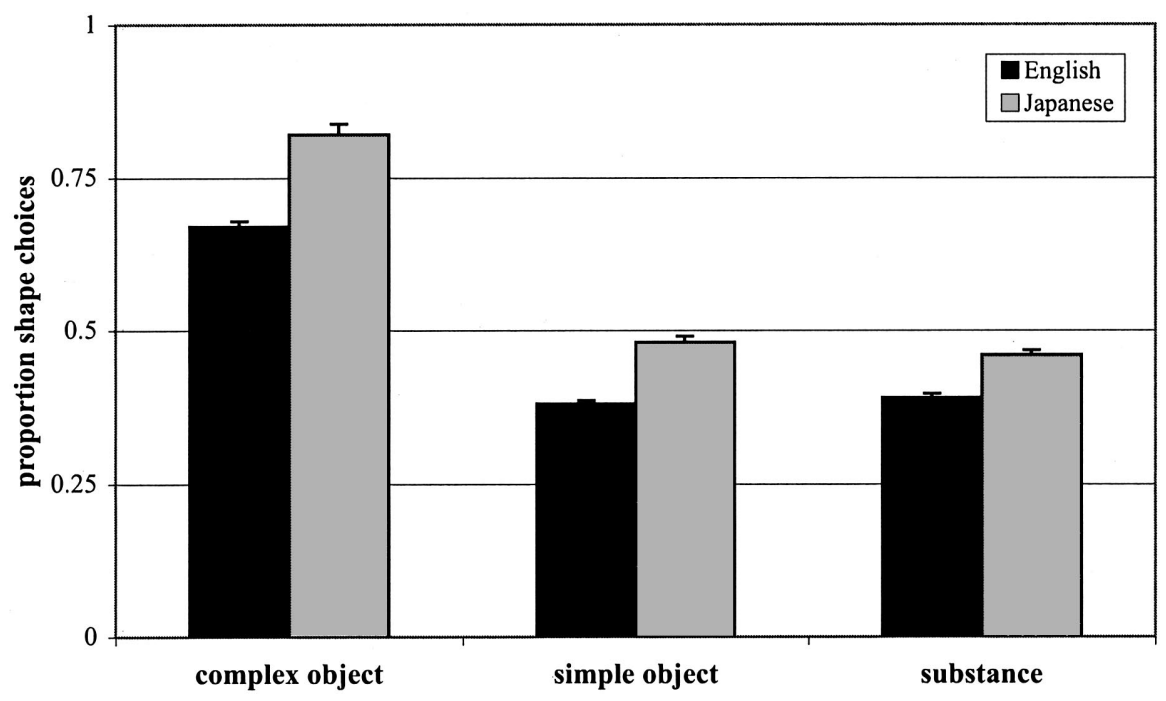

Figure 26. Mean proportion of shape choices by the networks trained on vocabularies incorporating the regularities among early Japanese nouns compared with networks trained on the regularities among early English nouns. Error bars represent standard error. 


\section{Method}

Procedure. To incorporate count-mass syntax into the networks' training set, we used judgments made by three native English speakers. In Samuelson and Smith's (1999) study, participants were asked to give a global judgment as to whether each noun was count, mass, neither, or both. However, because we wanted to model young children's performances, we wanted a more surface-level measure of these cues' correlations with category structure. Accordingly, we asked three native English speakers to answer four questions about each noun in the modeled set of English nouns (the 149 nouns used in Experiment 2). Adults answered the following questions for each of the nouns:

1. Cannot be used with much or less?

2. Can be used with many, several, or numerals?

3. Has a plural form?

4. Can be preceded with $a$ or $a n$ ?

Each noun was classified as count if all four questions were answered "yes," as mass if all four questions were answered "no," and otherwise as ambiguous. The analysis of these judgments revealed that, indeed, almost all solid, shape-based categories are named by count nouns $(95 \%)$ and that although most mass nouns do refer to material-based categories $(91 \%)$, only about half of the nonsolid, material-based categories are named by mass nouns $(47 \%)$.

Architecture. The networks used in this experiment had the same architecture as that used in Experiment 2 except for an added syntax layer as shown in Figure 27. The syntax layer had two units, one to represent count syntax and one for mass syntax.

Training and testing. The networks were trained on the English vocabulary of Experiment 8, incorporating the new count-mass syntax information. Testing was done as in Experiment 8; there was no syntax information given to the networks during testing.

\section{Results}

The results of the network simulations are shown in Figure 28. The proportions of shape choices predicted by each of the 10

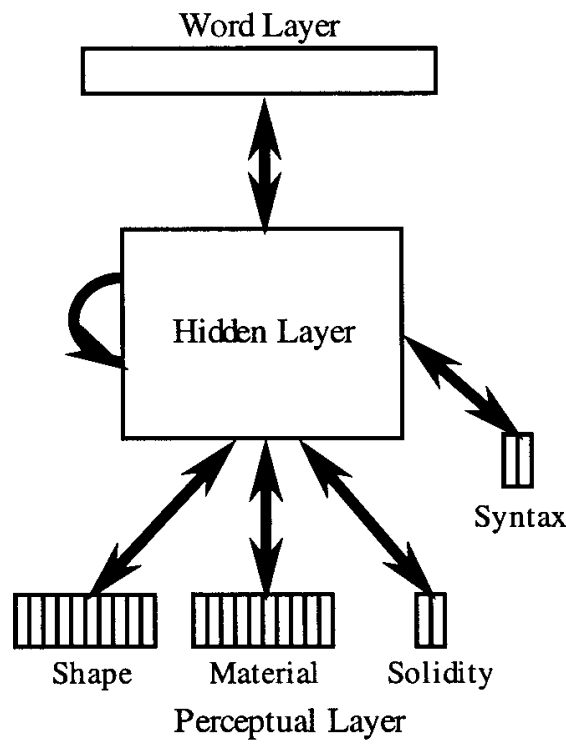

Figure 27. Architecture of the network used in Experiment 9. networks were submitted to a 3 (solidity) within-subject analysis of variance. The analysis revealed a main effect of solidity, $F(1$, $18)=306.211, p<.0001$. The networks were more likely to choose the shape match on the complex solid trials and on the simple solid trials than on the nonsolid trials. Further, for the complex solid trials and for the simple solid trials, the networks predicted more shape choices than expected by chance, $t(9)=$ $12.329, p<.0001$, for complex solids; $t(9)=4.014, p=.003$, for simple solids. For the nonsolid trials, the networks predicted less shape choices than expected by chance, $t(9)=-27.375, p<$ .0001 . Nine of the 10 networks showed this pattern; 1 network did not develop a shape bias for the simply shaped solids (and thus showed a pattern similar to the networks trained without syntax). Overall, then, the networks trained on the English-like vocabulary with count-mass syntax information performed like Englishspeaking children, treating simple solids like complex solids and differently from nonsolids. Thus, the connectionist networks trained on English with syntax were successful in modeling English-speaking children's behavior across the full range of shapes. The results of this experiment suggest that Englishspeaking children generalize names for simply shaped solid forms by shape because their language (at least the part of their language known to young children) often refers to simple solid forms using the same syntax used to name complexly shaped solid thingsthings overwhelmingly named by their shape.

\section{Summary of Experiments 7-9}

These final three experiments extend the associative learning account to cross-linguistic similarities and differences. There are three main results. First, the predicted relations between soliditynonsolidity and category structure in the early nouns were remarkably similar in both English and Japanese. Second, the statistical regularities in the early noun lexicon were enough to explain the similarities in English- and Japanese-speaking children's novel noun generalizations but not enough to explain the differences. Third, the differences in Japanese- and English-speaking children's noun extensions appear to be created by the additional correlations added by English count-mass syntax.

These results have implications for how we think about crosslinguistic universals and differences. They suggest that some universals might be the product of similar learning environments. In particular, Japanese- and English-speaking children's novel noun generalizations may be similar because both are products of generalizations over the nouns children know and because the nouns children know in the two languages have similar category structures. The results also suggest that the processes that make universal and language-specific differences may sometimes be the very same. That is, the underlying mechanism-associative learning and generalization by similarity - simulates both the similarities and the differences.

Quine (1960) once famously proposed that count-mass syntax actually created the abstract ideas of object and substance. Soja et al. (1992) argued that this proposal was wrong because Englishspeaking children generalized names for solids and nonsolids differently before they mastered count-mass syntax. Imai and Gentner (1997) also countered Quine by showing that Japanesespeaking children who spoke a language without any corresponding distinction also generalized names for solids and nonsolids 


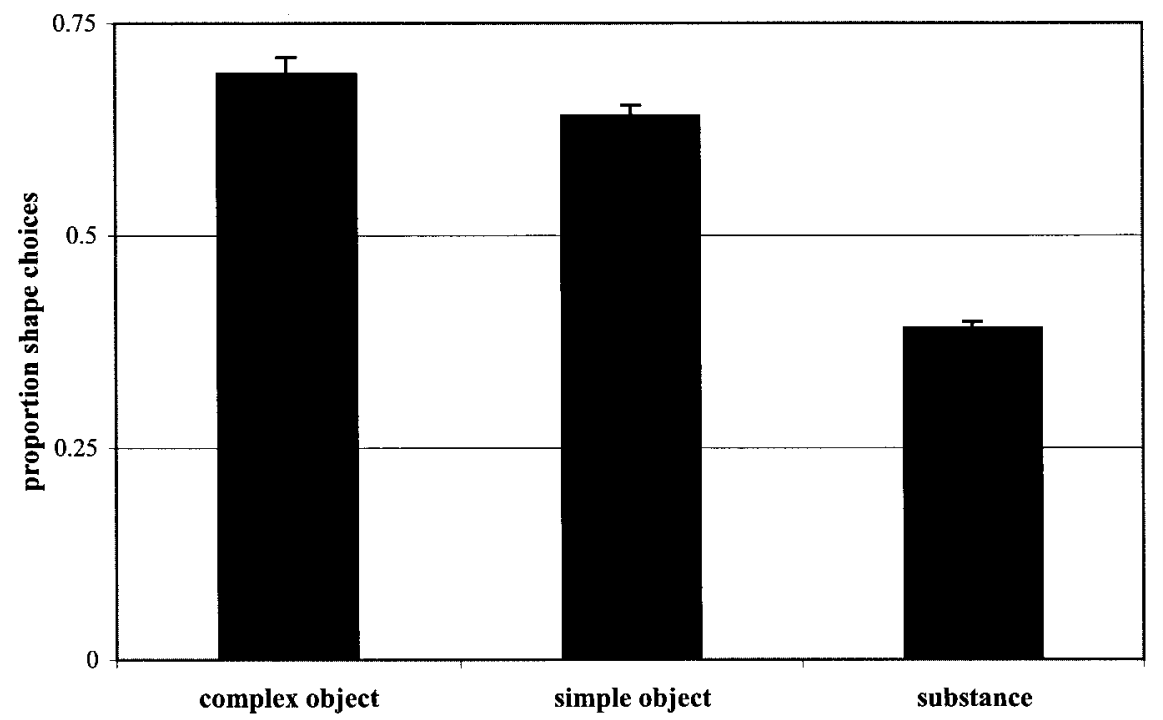

Figure 28. Mean proportion of shape choices predicted by the networks trained with additional count-mass syntax information. Error bars represent standard error.

differently. However, consistent with perhaps the germ of Quine's proposal, count-mass syntax does influence how English speakers categorize entities varying in their solidity and in their complexity (and constructedness) of shape. In this way, the specific language one learns does play a role in the development of distinct kinds.

However, the bigger lesson across the series of nine simulations and behavioral experiments is perhaps this: The distinctions that children make when generalizing a just-heard name for a single novel thing may be explained as generalizations over a complex pattern of correlations that include correlations among perceptual properties, lexical category structure, and other aspects of language. Within the reasonably large corpus of nouns that children know at 2 to 3 years of age, these regularities create higher order correlations that yield patterns of behavior suggestive of underlying concepts about different kinds. The present results show that these patterns may arise, at least in part, from no more than associations among learned instances.

\section{General Discussion}

One of the most remarkable aspects of children's early noun learning is how good they are at it; they need to hear the name of only a single instance to systematically generalize that name in ways that seem correct to adults. This skill is all the more remarkable because different kinds of things are organized into categories by different properties. The fact that children extend names for solid and nonsolid things in different ways thus suggests that they know something about solids and nonsolids as different kinds. Our starting question was how general processes of associative learning might contribute to this knowledge.

Associative learning is a good candidate for three reasons. First, it is a reasonably well-understood mechanism that children are known to possess. Second, it is a mechanism that is very good at internalizing and generalizing from noisy statistical regularities. Third, the nouns that children learn early present regularities that could be responsible for children's different name extensions for solids and nonsolids. The results of the simulations and behavioral experiments support this hypothesized role for learned associations in creating expectations about the category structures of different kinds. The simulations show that these general learning processes are capable of creating a generalized distinction between solids and nonsolids. Indeed, given the correlations in the early noun lexicon, the networks generalized that learning in a manner consistent with a rule that prohibits solids and nonsolids from being in the same category, as if solids and nonsolids are fundamentally different kinds. Young children show the same biases. The rulelike behavior of the networks, however, is not the result of a represented rule. Instead, their generalizations are the product of connection weights accrued over the pairings of names to specific instances. Consequently, the networks' generalizations are also graded and context-sensitive, as are the generalizations of young children. In what follows, we discuss the implications of these findings for the nature, development, and origin of children's knowledge about different kinds and the relation of these ideas to the theoretical constructs of object and substance.

\section{Origin of the Correlations}

The networks, and by implication the children, develop expectations about the category structures of solids and nonsolids as a consequence of the statistical regularities among already learned noun categories. This raises the theoretically potent question of why young children know the particular nouns that they do and why these nouns present the particular correlations they do. Why is the category structure of early learned nouns so similar across English and Japanese? It is interesting that it is not the case that English- and Japanese-speaking children know the very same lexical categories. The lists of early learned nouns-lists developed from large-scale normative studies of both languages-indicate many differences in particular lexical categories. For example, many of the nonsolid categories are food items, and there are many differences between the two languages in food categories. Still, in 
both languages, names for solid things are more frequent than names for nonsolids, and there are more shape-based categories than material-based categories. Why do early vocabularies have the structure that they do?

The nouns children know are the developmental product of the nouns they hear and the internal biases that children bring to the learning task. So it might be argued that the regularities that are seen among early nouns are primarily the product of biases that already exist in children rather than, as we propose, the result of learning the early lexicon. We consider this issue, first taking a narrow perspective that argues against preexisting biases by reviewing empirical evidence on the development of novel noun generalizations and evidence on the nouns children hear. We then take a broader perspective and consider the theoretically more difficult question of why early noun vocabularies have the structures they do and what this means about the origins of distinctions between kinds.

The extant evidence provides little support for the idea that the shape and material biases precede word learning. Children do not show evidence of the shape bias in the novel noun generalization task until they know a considerable number of nouns (e.g., Samuelson \& Smith, 1999). Further, children's earliest noun vocabularies (under 25 nouns) show little evidence of solids being mostly named by shape and nonsolids being mostly named by material (Smith, 1995). Instead, these regularities emerge as early vocabularies grow. Moreover, the nouns individual children know appear to closely match the nouns they hear (Huttenlocher et al., 1991), and the regularities among the nouns children know appear to match well the regularities among the nouns they hear (Sandhofer et al., 2001). Further, the data from the present experiments strongly suggest that children learn the correlations they exploit in the novel noun generalization task - the overarching one that solid things are named by shape and nonsolid things by material, as well as correlations concerning the constructedness of shape and, for English-speakers, count-mass syntax. All of this evidence points to children's biases in the novel noun generalization task as products of the correlational structure of early noun categories rather than the correlational structure of early vocabularies being the product of internal biases.

However, there is potentially contradictory evidence to this conclusion. Lucy (1992) presented evidence from speakers of Yucatec Mayan that might be interpreted in terms of a shape bias that is independent of lexical categories. Yucatec presents a relevant case because nouns in this language do not refer to discrete, countable entities as do English names; instead, all nouns in Yucatec are quantificationally neutral, as are mass nouns in English. So untz' iit kib is used to refer to one candle, with untz'iit meaning something like "one long thin" and kib typically translated as meaning "wax." In everyday speech the classifier-one long thin-is often omitted. Thus, this is a language that seems to point to material categories with little attention to shape. Consistent with this idea, adult speakers of Yucatec classify solid objects by material. Given that the nouns in Yucatec refer to materialbased categories, this fact may be taken as support for the idea that the lexical categories one learns create the distinctions one makes. However, the developmental evidence from learners of Yucatec suggests a strikingly contradictory conclusion. Speakers of $\mathrm{Yu}-$ catec do not show a material bias for objects until they are older than 9 years. Children between 7 and 9 years show a shape bias (evidence from younger children has not been reported). This could mean that children have a strong innate bias to categorize solid objects by their shape and that it takes many years of learning a contrary language to override this bias.

There is, however, an alternative account. In everyday usage, Yucatec speakers use their nouns to refer to functional categories of things; things referred to by the same name thus typically do have the same shape (even though the noun means the material). The early shape bias in Yucatec Mayan may reflect children's experiences with how nouns are actually used by speakers of the language in everyday context. That is, the correlations that matter for young children are not those that reside in the formal analysis of the language as a whole; rather, the correlations that matter for young children are those that exist between individual utterances of nouns and the individual entities to which they refer. If in the everyday lives of children learning Yucatec, kib and other nouns are used to refer to objects with particular characteristic shapes, then children will learn to attend to the shapes of objects. Perhaps, after many years of learning their language and broader experiences with nouns that include cases of these same nouns referring to entities similar in material only, they may shift their attention to material.

These arguments still leave unexplained the question of why early nouns have the structure they do, why people (at least statistically) refer to categories of solid things by shape and categories of nonsolid things by material. There are two likely causes. One is the physical structure of the world. Solid things have invariant shapes and invariant materials over movement and many other transformations; in contrast, nonsolid things have transient shapes but invariant material. It makes sense to refer to things by their stable properties. In addition, there are, as a consequence of physics, shape-propensity correlations and material-propensity correlations. For example, certain kinds of shapes can with proper movement swim more easily than other kinds, certain kinds of shapes but not others afford carrying other things, and certain kinds of materials but not others can soak up liquids. The second likely cause is human psychology and biology. For example, the visual system may be tuned to attend to the invariant and nonaccidental properties of rigid shapes (Biederman, 1987; Spelke, 1990; Wisniewski, Lamb, \& Middleton, 2003). Further, and perhaps more important, the property-propensity correlations in the world are important to how people use and react to things in the world. In particular, the functional properties of solid things may depend mostly on their shape, whereas the functional properties of nonsolid things may depend mostly on their material (see Gelman \& Bloom, 2000; Leyton, 1992; Samuelson \& Smith, 2000). These facts about the physical world and human psychology may create the statistical regularities in the early noun lexicon. Languages must, after all, evolve words that match human psychological needs. But still, it may be the statistical regularities in the early noun lexicon that create-via ordinary processes of associative learning — children's generalized expectations about the category structures of different kinds.

\section{A Web of Correlations}

Although we began this article with the solidity-nonsolidity distinction and although solidity is a strong predictor of category structure, our full pattern of findings makes clear that children's 
knowledge about different kinds is not simply knowledge about the predictive relation between solidity and category structure. Instead, it is also about the overlapping correlations among solidity, the constructedness-complexity of shape, language, and lexical category structure. Shape constructedness appears to play a particularly strong role. This is predicted by our analysis of the similarity space, that solid things can take more varied shapes, including ones with many parts (and thus more evidence of their nonaccidental nature), and by the correlations in the early lexicon. Gelman and Bloom (2000) and Prasada et al. (2002) have also argued on other grounds that nonaccidental shapes are crucial to children's and adults' conceptualizations of artifactual objects as being "designed" for some purpose. Although our results concern children's extension of category names by perceptual properties (and not their reasoning about origins), they support the idea that kind of shape is a crucial factor in children's developing knowledge about kinds.

Our results also show that syntax is part of the correlational mix. In the early lexicon, the syntactic frames in which nouns are presented correlate both with the perceptual properties of things and with their category structure. Our simulations of Imai and Gentner's (1997) results illustrate how those correlated syntactic cues interact with correlations between perceptual properties such as solidity, kind of shape, and category structure. Our simulations suggest that the count syntax correlations available to English speakers push them to view simply shaped solids as more like complexly shaped solids than like nonsolids. The implication is that perceptual cues such as the deformation of shape when touched (our definition of solidity in the adult judgments) have more weight for English speakers than for Japanese speakers because they correlate with syntax, whereas perceptual cues about the constructedness of shape may have more weight for Japanese speakers. This is a testable prediction for future work. The critical point is that by our account, all of these factors-perceptual properties, syntactic frame, and category structure-jointly and through the same mechanisms create children's generalized expectations about how solid and nonsolid things are named.

Learned associations, like correlations, are bidirectional. Thus, if solidity predicts shape categories, then shape categories predict solidity, and if count-noun syntax predicts constructed shapes, then constructedness of shape predicts count syntax. Our experiments present some evidence for this bidirectionality in that the solidity of the named entity predicts a category of instances of the same solidity, as well as instances of the same shape, and constructedness of shape predicts a category organized by shape. Hall (1996) and Prasada et al. (2002) have also reported evidence of correlations in both directions. Specifically, they reported that solidity, constructedness of shape, and the category relevance of shape all predict count-noun syntax. Bidirectionality is computationally important because it means that overlapping but noisy correlations build on and mutually reinforce each other (see Yoshida \& Smith, 2003a). Bidirectionality is developmentally important because it means that cues codevelop. By such a model, attention to solidity is not a prerequisite to learning about shape nor is learning about shape a prerequisite to learning about solidity. Rather, the predictive relation between the two and the increased attention that predictive relation engenders will codevelop.

These ideas have relevance beyond the distinction between categories of solid and nonsolid things. There are correlations in the learning environment beyond those that partition solid and nonsolid things. Recent studies of children's categories (e.g., Lavin \& Hall, 2001; Yoshida \& Smith, 2003a, 2003c) as well as of adults' categories (e.g., McRae et al., 1997) suggest that correlations between perceptual properties, actions, functions, and category structure may underlie partitions between such different kinds as foods, tools, vehicles, and animals. The present account suggests that children should learn about these distinctions-and be able to extend that learning to novel instances-as their noun vocabularies expand and as these associations accrue. In brief, the processes studied here may be relevant to explaining children's expectations about a wide variety of kinds. The power of these potential explanations derives from the incorporation of multiple and bidirectionally interconnected correlations of various strengths. In the present case, we found that for English-speaking children, solidity was correlated with kind of shape, with syntax, and with category structure. Kind of shape, in turn, was correlated with syntax, with category structure, and with solidity. Children's expectations, then, about the different kinds studied here are dependent on a web of correlations among a number of properties of language, of objects, and of object categories. This, we suggest, may be the very nature of knowledge about different kinds.

The fact that the knowledge is at root correlational also means that these global patterns will be context-sensitive, varying adaptively to meet specific tasks and the specific learning environment. In this way, children's knowledge about different kinds may be characterized as a system of soft regularities, much as has been proposed for English spelling to sound correspondences (e.g., Seidenberg \& McClelland, 1989) or their knowledge of morphology (e.g., McClelland \& Patterson, 2002).

\section{Networks and Children}

All modeling is simplification, and the particular implications that derive from our simulations depend on the significance placed on connectionist modeling and, more broadly, on what one takes to be the role of modeling in theory and experimentation. One could take a narrow view and think of these networks as computational devices that simply measure the regularities in their input. If so, the contributions of the simulations lie in three areas. First, our simulations show that there are regularities among early lexical categories - perceptual information, syntactic information, and withincategory similarities-that are sufficient to create generalized expectations about how to categorize even novel things. Second, they provide a computational tool for generating predictions from a complicated, messy, noisy mass of correlations. Finally, our experiments with simple neural networks show the plausibility of learning second-order generalizations from specific individual instantiations of the first-order categories.

We prefer a broader view, that the assumptions about mechanisms that underlie these simple statistical learners may also be psychologically (and biologically) appropriate descriptions of the learning process. According to this view, the simulations have important implications for the origin, nature, and consequences of children's knowledge about naming solids and nonsolids. For example, the associative learning account offers insights into how children become "smart" word learners. The networks are generalpurpose learning devices. They begin with built-in sensitivities to particular perceptual properties but with no prespecified biases to 
attend to some properties in particular contexts and to others in a different context. However, with learning, the networks become fine-tuned, internalizing the correlations present in their environment. A learner endowed with such ability would be very powerful indeed, as it would be able to tweak itself and adapt to the language learned and to the specific category at hand. This implies that as long as there are regularities that can be exploited by the learner to simplify its learning task, these regularities do not have to be instantiated in the minds of learners prior to learning.

The assumptions behind the simulations also have implications for explaining the developmental trend in the novel noun generalization task. The theoretical work reported in this article is admittedly limited in this regard as it focused on the statistical match between the nouns known by 2 - to 3 -year-olds and their novel noun generalizations. However, there are possible extensions of the present account that may prove informative as to why the developmental trend looks as it does. For example, the networks' distinction between solids and nonsolids requires the formation of higher order correlations between cues (perceptual properties such as solidity or syntactic frame) and category structure. Category structure, in turn, is a lower order generalization over associations between particular names (e.g., "ball") and particular specific things (e.g., a round rubber yellow ball, a wooden croquet ball, or a large beach ball). It is interesting that the formation of higher order correlations may depend systematically on the number and range of lower order correlations, and this may explain why very young word learners (12- to 18-month-olds) conservatively generalize novel names for novel things, restricting the name to nearly identical things (e.g., Woodward et al., 1994), and it may also explain why a generalized shape bias emerges sooner and stronger than a generalized material bias. The dependence of these higher order correlations on lower order lexical categories also raises the question of whether and how lexical learning per se (vs. other forms of category learning) might play a computationally important role in children's developing knowledge about kinds. These ideas also need to be pursued in future work.

\section{Associations and Concepts of Object and Substance}

We have offered an explanation of children's generalized expectations about how names map to categories of novel solid and novel nonsolid things, suggesting that these expectations are the product of a web of correlations and the higher order generalizations they afford, learned as children learn nouns. What does this explanation-and the results that support it-imply about the theoretical constructs of object and substance concepts? One possibility is that the theoretical constructs of object and substance are merely summary terms for the kinds of associations studied here. Correlational learning may underlie behaviors suggestive of a psychological ontology, that is, representations that specify the fundamental nature of different kinds. This seems particularly plausible if the bundles of correlations that create mature knowledge include more than the perceptual properties. McRae et al. (1997) provided an example of this idea. They modeled adult judgments of semantic relatedness with an associative theory in which the features are perceptual properties (e.g., shape, moves on own) and relational roles (e.g., used to carry water, friendly). Extending the theory in this way may allow us to explain phenom- ena generally interpreted as contrary to the associative learning account.

For example, Prasada et al. (2002) cleverly showed that whether adults construe an entity as an object or a substance depends critically on their beliefs about the importance of shape rather than on the perceptual property of the shape itself. Adults were presented with an accidental bloblike form. When they were presented with several exact replications of that accidental shape, the adults took the shape as nonaccidental and therefore important to the lexical category. This result, at first glance, might be interpreted as showing that adults' reasoning about object and substance categories involves different mechanisms than the correlational learning studied here, as we showed that accidental shapes are correlated with material categories. However, the main difference may not be the mechanism - or the nature of underlying knowledge - but in the correlations. The correlational structure that generates adults' judgments may include more than associations among solidity, shape, and material; specifically, the correlational structure may include associations among words, experiences with instances of exactly the same shape, and means of manufacturing things. Indeed, with development, concrete perceptual properties-such as deformation of shape by touch-may become less important as verbal descriptions, event structures, or function becomes the more pervasive and thus more predictive cues. Nonetheless, if the underlying knowledge is associative, then adults should still show graded and context-sensitive patterns of judgments that are influenced by solidity and constructedness of shape. The relevance of these perceptual properties is expected to remain because these correlations so strongly characterize a portion of the adult noun lexicon, the early learned names for concrete objects and substances.

A contrasting position can also be argued. Children's novel noun extensions for solids and nonsolids (and our associative account of those data) may be only remotely connected to concepts of object and substance. One starting point for this counterargument is that most of the data typically taken by others as indicating object and substance concepts are not about solidity and nonsolidity but are, instead, about countability. That is, the objectsubstance distinction is usually defined as being about whether an entity is conceptualized as discrete and thus countable or whether it is conceptualized as a continuous quantity (e.g., Gordon, 1985; Lakoff \& Johnson, 1980; Lenat \& Guha, 1990; Pelletier, 1979). As so defined, this distinction is important to reasoning about quantities, to grammatical class in many languages, and to a variety of inferences that one can make about different kinds. By this definition, objects may be solid or nonsolid (e.g., chairs and bubbles) or even abstract (e.g., ideas), and substances may be solid, nonsolid, or abstract (e.g., wood, water, and justice). One real possibility, then, is that children's different name extensions for solids and nonsolids in the novel noun generalization task-and the knowledge and processes that underlie those performances-have little to do with their understanding of what is countable or not.

Whereas children's generalized expectations about naming solids and nonsolids may derive from learned associations, children's concepts of object and substance may have their origins in infants' concepts of number. Indeed, recent work on infant number concepts suggests that before word learning, infants distinguish cohesive (typically solid) things and noncohesive (typically nonsolid) things as countable versus noncountable (see Chiang \& Wynn, 
2000; Feigenson, Carey, \& Spelke, 2002; Huntley-Fenner et al., 2002; Xu, 1997; see also van Marle \& Scholl, 2003). Still, one reason developmentalists have been so interested in children's different name extensions for solids and nonsolids is the likely relevance of this distinction to concepts of object and substance. Solidity seems an ideal expression of objectness in that solid things are discrete with cohesive, bounded shapes. Likewise, nonsolidity seems an ideal expression of substances because nonsolids are noncohesive masses with stable materials but transient forms. It is clear that an important theoretical problem to be resolved is the relation between children's use of perceptual cues to category structure in the novel noun generalization task, infants' attention to the number of cohesive but not noncohesive things, and developing knowledge about objects and substances.

\section{Conclusion}

The idea that differences in correlational structure create people's knowledge about different kinds of things has been offered by others. For example, Gelman (1988) and Keil (1989) argued that the distributional patterns of correlated features differ across artifacts and living things. McRae et al. (1997) provided evidence showing that features within a single category of animate things are more densely intercorrelated and less variable than features within individual categories of inanimate things. Such correlational differences have also been offered as explanations for category-specific deficits in individuals with brain damage (Devlin, Gonnerman, Andersen, \& Seidenberg, 1998; Farah \& McClelland, 1991). Patients have been described with deficits that suggest distinct systems for animals, plants, artifacts, and also foods. It has been suggested that these domains are neurally segregated because they depend on different sets of intercorrelated properties. All of these ideas clearly are consistent with the present demonstration that correlated properties may create young children's expectations about how solids and nonsolids are named. However, the present work goes beyond these prior suggestions in at least three ways.

First, we show that the correlational differences that characterize individual categories create higher level correlations between category structure and particular perceptual properties such as solidity and type of shape. These higher level correlations, in turn, create higher level categories that transcend the specific properties of specific things. Thus, the learner has expectations about category structure for things not yet encountered, that is, generalized expectations about solid and nonsolid things. Second, we place the origins of these generalized expectations in the correlational structures of early learned noun categories. Third, we show how unbiased learners become biased learners. The networks used in these simulations use a simple local correlational learning rule. At the start of learning, this is all the network has, and it is a relatively unbiased learner. But with each word the network learns, it becomes more biased, developing expectations that are both increasingly general and increasingly fine-tuned about the correlations that matter for different kinds. The network as a whole, its correlational learning rule, and its past learning are the model of the learner. From this perspective, the learner and the learning process change with each word learned. Thus, children may start with a simple mechanism and as relatively unbiased learners. However, through engagement with the world, learners become smart; they know that there are different kinds of things in the world that are categorized in different ways.

\section{References}

Biederman, I. (1987). Recognition by components: A theory of human image understanding. Psychological Review, 94, 115-147.

Bloom, P. (1996). Intention, history and artifact concepts. Cognition, 60, $1-29$.

Bloom, P. (2000). How children learn the meanings of words. Cambridge, MA: MIT Press.

Booth, A., \& Waxman, S. (2002a). Object names and object functions serve as cues to categories for infants. Developmental Psychology, 38, 948-957.

Booth, A., \& Waxman, S. (2002b). Word learning is "smart": Evidence that conceptual information affects preschoolers' extension of novel words. Cognition, 84, B11-B22.

Booth, A., \& Waxman, S. (2003). Mapping words to the world in infancy: Infants' expectations for count nouns and adjectives. Journal of Cognition \& Development, 4, 357-381.

Burger, J., \& Prasada, S. (1997). Quantification of solid and nonsolid entities: The role of perceived arbitrariness of structure. In E. Hughes, M. Hughes, \& A. Greenhill (Eds.), Proceedings of the Boston University Conference on Child Language (pp. 36-44). Boston: Cascadilla Press.

Bushnell, E. W. (1982). Visual-tactual knowledge in 8-, 9.5-, and 11month-old infants. Infant Behavior and Development, 5, 63-75.

Bushnell, E. W., \& Boudreau, J. P. (1991). The development of haptic perception during infancy. In M. A. Heller \& W. Schiff (Eds.), The psychology of touch (pp. 139-161). Hillsdale, NJ: Erlbaum.

Chiang, W., \& Wynn, K. (2000). Infants' tracking of objects and collections. Cognition, 77, 169-195.

Christiansen, M. H., \& Chater, N. (2001). Connectionist psycholinguistics: Capturing the empirical data. Trends in Cognitive Sciences, 5, 82-88.

Colunga, E., \& Smith, L. B. (2003). The emergence of abstract ideas: Evidence from networks and babies. Philosophical Transactions by the Royal Society, 358, 1205-1214.

Colunga, E., \& Smith, L. B. (2004). Correlation versus prediction in children's word learning: Cross-linguistic evidence and simulations. Manuscript submitted for publication.

Devlin, J. T., Gonnerman, L. M., Andersen, E. S., \& Seidenberg, M. S. (1998). Category-specific semantic deficits in focal and widespread brain damage: A computational account. Journal of Cognitive Neuroscience, 10(1), 77-94.

Dickinson, D. K. (1988). Learning names for materials: Factors constraining and limiting hypotheses about word meaning. Cognitive Development, 3, 15-35.

Diesendruck, G., \& Bloom, P. (2003). How specific is the shape bias? Child Development, 74, 168-178.

Diesendruck, G., Markson, L., Bloom, P. (2003). Children's reliance on creator's intent in extending names for artifacts. Psychological Science, 14, $164-168$.

Farah, M. J., \& McClelland, J. L. (1991). A computational model of semantic memory impairment: Modality specificity and emergent category specificity. Journal of Experimental Psychology: General, 120, 339-357.

Farkas, I., \& Li, P. (2001). A self-organizing neural network model of the acquisition of word meaning. In M. E. Altmann \& A. Cleeremans (Eds.), Proceedings of the 2001 Fourth International Conference on Cognitive Modeling (pp. 67-72). Mahwah, NJ: Erlbaum.

Feigenson, L., Carey, S., \& Spelke, E. (2002). Infants' discrimination of number versus continuous extent. Cognitive Psychology, 44, 33-66.

Fenson, L., Dale, P., Reznick, J. S., Thal, D., Bates, E., Hartung, J., et al. (1993). The Macarthur Communicative Development Inventory: User's guide and technical manual. San Diego, CA: Singular Publishing Group. 
Gathercole, V. (1997). The linguistic mass/count distinction as an indicator of referent categorization in monolingual and bilingual children. Child Development, 68, 832-842.

Gathercole, V. C. M., Cramer, L., Somerville, S. C., \& Haar, M. J. O. D. (1995). Ontological categories and function: Acquisition of new names. Cognitive Development, 10, 225-251.

Gelman, S. A. (1988). The development of induction within natural kind and artifact categories. Cognitive Psychology, 20, 65-95.

Gelman, S. A., \& Bloom, P. (2000). Young children are sensitive to how an object was created when deciding what to name it. Cognition, 76, 91-103.

Gibson, E. J., Owsley, C. J., Walker, A., \& Megaw-Nyce, J. (1979). Development of the perception of invariants: Substance and shape. Perception, 8, 609-619.

Gibson, J. J. (1979). The ecological approach to visual perception. Hillsdale, NJ: Erlbaum.

Golinkoff, R., Hirsh-Pasek, K., Bloom, L., Smith, L. B., Woodward, A., Akhtar, N., et al. (2000). Becoming a word learner: A debate on lexical acquisition. London: Oxford University Press.

Goodman, N. (1983). Fact, fiction, and forecast. New York: Bobbs Merrill. (Original work published 1955)

Gordon, P. (1985). Evaluating the semantic categories hypothesis: The case of the count/mass distinction. Cognition, 20, 209-242.

Gordon, P. (1988). Count/mass category acquisition: Distributional distinctions in children's speech. Journal of Child Language, 15, 109-128.

Hall, D. G. (1994). How children learn common nouns and proper names: A review of the experimental evidence. In J. Macnamara \& G. Reyes (Eds.), The logical foundations of cognition: Vancouver, British Columbia, Canada, studies in cognitive science (Vol. 4, pp. 212-240). London: Oxford University Press.

Hall, D. G. (1996). Naming solids and nonsolids: Children's default construals. Cognitive Development, 11, 229-264.

Hollich, G. J., Hirsh-Pasek, K., Golinkoff, R. M., Brand, R. J., Brown, E., Chung, H. L., et al. (2000). Breaking the language barrier: An emergentist coalition model for the origins of word learning. Monographs of the Society for Research in Child Development, 65(3).

Hopfield, J. J. (1982). Neural networks and physical systems with emergent collective computational abilities. Proceedings of the National Academy of Sciences, USA, 79, 2554-2558.

Hopfield, J. (1984). Neurons with graded response have collective computational properties like those of two-state neurons. Proceedings of the National Academy of Sciences, USA, 81, 3088-3092.

Huntley-Fenner, G., Carey, S., \& Solimando, A. (2002). Objects are individuals but stuff does not count: Perceived rigidity and cohesiveness influence infants' representations of small groups of discrete entities. Cognition, 85, 203-221.

Huttenlocher, J., Haight, W., Bryk, A., Seltzer, M., \& Lyons, T. (1991). Early vocabulary growth: Relation to language, input, and gender. Developmental Psychology, 27, 236-248.

Imai, M., \& Gentner, D. (1997). A cross-linguistic study of early word meaning: Universal ontology and linguistic influence. Cognition, 62, $169-200$

Jones, S. S., Smith, L. B., \& Landau, B. (1991). Object properties and knowledge in early lexical learning. Child Development, 62, 499-516.

Keil, F. C. (1989). Concepts, kinds, and cognitive development. Cambridge, MA: MIT Press.

Keil, F. (1994). The birth and nurturance of concepts by domains: The origins of concepts of living things. In L. Hirschfeld \& S. Gelman (Eds.), Mapping the mind: Domain specificity in cognition and culture (pp. 234-254). New York: Cambridge University Press.

Kemler Nelson, D. G., Frankenfield, A., Morris, C., \& Blair, E. (2000). Young children's use of functional information to categorize artifacts: Three factors that matter. Cognition, 77, 133-168.

Kobayashi, H. (1997). The role of actions in making inferences about the shape and material of solid objects among 2-year-old children. Cognition, 63, 251-269.

Lakoff, G., \& Johnson, M. (1980). Metaphors we live by. Chicago: University of Chicago Press.

Landau, B., Smith, L. B., \& Jones, S. S. (1988). The importance of shape in early lexical learning. Cognitive Development, 3, 299-321.

Lavin, T., \& Hall, D. G. (2001). Domain effects in lexical development: Learning words for foods and toys. Cognitive Development, 16, 929950 .

Lenat, D., \& Guha, R. V. (1990). Building large knowledge based systems: Representation and inference in the Cyc Project. New York: AddisonWesley.

Levy, Y. (1988). On the early learning of formal grammatical systems: Evidence from studies of the acquisition of gender and countability Journal of Child Language, 15, 179-187.

Leyton, M. (1992). Symmetry, causality, mind. Cambridge, MA: MIT Press.

Luce, R. D. (1963). Detection and recognition. In R. D. Luce, R. R. Bush, \& E. Galanter (Eds.), Handbook of mathematical psychology (pp. 1-41). New York: Wiley.

Lucy, J. A. (1992). Grammatical categories and cognition: A case study of the linguistic relativity hypothesis. Cambridge, England: Cambridge University Press.

Lucy, J. A., \& Gaskins, S. (2001). Grammatical categories and the development of classification preferences: A comparative approach. In M. Bowerman \& S. C. Levinson (Eds.), Language acquisition and conceptual development (pp. 257-283). Cambridge, England: Cambridge University Press.

Marcus, G. (2001). The algebraic mind: Integrating connectionism and cognitive science. Cambridge, MA: Bradford Books.

Mash, C., Quinn, P. C., Dobson, V., \& Narter, D. B. (1998). Global influences on the development of spatial and object perceptual categorization abilities: Evidence from preterm infants. Developmental Science, 1(1), 85-102.

McClelland, J., \& Patterson, K. (2002). Rules or connections in past-tense inflections: What does the evidence rule out? Trends in Cognitive Sciences, 6, 465-472.

McClelland, J. L., \& Rumelhart, D. E. (1986). Parallel distributed processing: Explorations in the microstructure of cognition (Vol. 2). Cambridge, MA: MIT Press.

McPherson, L. (1991). A little goes a long way: Evidence for a perceptual basis of learning for the noun categories COUNT and MASS. Journal of Child Language, 18, 315-338.

McRae, K., de Sa, V. R., \& Seidenberg, M. S. (1997). On the nature and scope of featural representations of word meaning. Journal of Experimental Psychology: General, 126, 99-130.

Moss, H. E., Hare, M. L., Day, P., \& Tyler, L. K. (1994). A distributed memory model of the associative boost in semantic priming. Connection Science: Journal of Neural Computing, Artificial Intelligence and Cognitive Research, 6, 413-427.

Movellan, J. (1990). Contrastive Hebbian learning in the continuous Hopfield model. In D. Touretzky, J. Elman, T. Sejnowski, \& G. Hinton (Eds.), Proceedings of the 1990 Connectionist Models Summer School (pp. 10-17). San Mateo, CA: Morgan Kaufmann.

Murphy, G. L., \& Wisniewski, E. J. (1989). Feature correlations in conceptual representations. In G. Tiberghien (Ed.), Advances in cognitive science: Vol. 2. Theory and applications (pp. 23-45). Chichester, England: Ellis Horwood.

Ogura, T., \& Watamaki, T. (1997). Japanese Communicative Developmental Inventories: User's guide and technical manual. San Diego, CA: Singular.

Ogura, T., Yamashita, Y., Murase, T., \& Dale, P. S. (1993). Some findings from the Japanese Early Communicative Developmental Inventories. Memoirs of the Faculty of Education, Shimane University, 27, 26-38. 
Pelletier, F. J. (1979). Mass terms: Some philosophical problems. Dordrecht, the Netherlands: Reidel.

Prasada, S., Ferenz, K., \& Haskell, T. (2002). Conceiving of entities as objects and as stuff. Cognition, 83, 141-165.

Quine, W. V. O. (1960). Word and object. Cambridge, MA: MIT Press.

Regier, T., \& Carlson, L. A. (2001). Grounding spatial language in perception: An empirical and computational investigation. Journal of Experimental Psychology: General, 130, 273-298.

Rosch, E. (1973a). Natural categories. Cognitive Psychology, 7, 573-605.

Rosch, E. (1973b). On the internal structure of perceptual and semantic categories. In T. E. Moore (Ed.), Cognitive development and the acquisition of language (pp. 111-144). New York: Academic Press.

Roy, D., \& Pentland, A. (2002). Learning words from sights and sounds: A computational model. Cognitive Science, 26, 113-146.

Rumelhart, D., \& McClelland, J. (1986). Parallel distributed processing: Explorations in the microstructure of cognition (Vol. 1). Cambridge, MA: MIT Press.

Samuelson, L. (2002). Statistical regularities in vocabulary guide language acquisition in connectionist models and 15-20-month-olds. Developmental Psychology, 38, 1016-1037.

Samuelson, L., \& Smith, L. B. (1999). Early noun vocabularies: Do ontology, category structure and syntax correspond? Cognition, 73, $1-33$.

Samuelson, L. K., \& Smith, L. B. (2000). Children's attention to rigid and deformable shape in naming and non-naming tasks. Child Development, $71,1555-1570$

Sandhofer, C. M., Smith, L. B., \& Luo, J. (2001). Counting nouns and verbs in the input: Differential frequencies, different kind of learning? Journal of Child Language, 27, 561-585.

Schyns, P. G. (1991). A modular neural network model of concept acquisition. Cognitive Science, 15, 461-508.

Seidenberg, M., \& McClelland, J. (1989). A distributed, developmental model of word recognition and naming. Psychological Review, 96, 523-568.

Shipley, E. F. (1993). Categories, hierarchies, and induction. In D. L. Medin (Ed.), The psychology of learning and motivation: Advances in research and theory (Vol. 30, pp. 265-301). San Diego, CA: Academic Press.

Shipley, E. F. (2000). Children's categorization of objects: The relevance of behavior, surface appearance, and insides. In B. Landau, J. Sabini, J. Jonides, \& E. Newport (Eds.), Perception, cognition and language: Essays in honor of Henry and Lila Gleitman (pp. 69-86). Cambridge, MA: MIT Press.

Smith, L. B. (1995). Self-organizing processes in learning to learn words: Development is not induction. In C. Nelson (Ed.), Basic and applied perspectives on learning, cognition, and development (Vol. 28, pp. 1-32). Mahwah, NJ: Erlbaum.

Smith, L. B., Colunga, E., \& Yoshida, H. (2003). Making an ontology: Cross-linguistic evidence. In D. Rakison \& L. Oakes (Eds.), Early category and concept development: Making sense of the blooming, buzzing confusion (pp. 275-302). London: Oxford University Press.

Smith, L. B., Gasser, M., \& Sandhofer, C. (1997). Learning to talk about the properties of objects: A network model of the development of dimensions. In R. L. Goldstone, D. L. Medin, \& P. G. Schyns (Eds.), The psychology of learning and motivation: Perceptual learning (Vol. 36, pp. 220-255). San Diego, CA: Academic Press.

Soja, N. N. (1992). Inferences about the meaning of nouns: The relationship between perception and syntax. Cognitive Development, 7, 29-45.

Soja, N. N., Carey, S., \& Spelke, E. S. (1991). Ontological categories guide young children's inductions of word meaning: Object terms and substance terms. Cognition, 38, 179-211.

Soja, N. N., Carey, S., \& Spelke, E. (1992). Perception, ontology, and word meaning. Cognition, 45, 101-107.

Spelke, E. S. (1990). Origins of visual knowledge. In D. N. Osherson \& S. M. Kosslyn (Eds.), Visual cognition and action: An invitation to cognitive science (pp. 99-127). Cambridge, MA: MIT Press.

Spelke, E. S., Breinlinger, K., Macomber, J., \& Jacobson, K. (1992). Origins of knowledge. Psychological Review, 99, 605-632.

Spelke, E., \& Hespos, S. (2001). Continuity, competence, and the object concept. In E. Dupoux (Ed.), Language, brain, and cognitive development: Essays in honor of Jacques Mehler (pp. 325-340). Cambridge, MA: MIT Press.

Subrahmanyam, K., Landau, B., \& Gelman, R. (1999). Shape, material, and syntax: Interacting forces in children's learning in novel words for objects and substances. Language \& Cognitive Processes, 14, 249-281.

Thornton, C. (2000). Truth from trash: How learning makes sense. Cambridge, MA: MIT Press.

van Marle, K., \& Scholl, B. (2003). Attentive tracking of objects versus substances. Psychological Science, 14, 498-504.

Wattenmaker, W. D. (1991). Learning modes, feature correlations, and memory-based categorization. Journal of Experimental Psychology: Learning, Memory, and Cognition, 17, 203-222.

Wattenmaker, W. D. (1993). Incidental concept learning, feature frequency, and correlated properties. Journal of Experimental Psychology: Learning, Memory, and Cognition, 19, 908-923.

Waxman, S., \& Markow, D. (1995). Words as invitations to form categories: Evidence from 12- to 13-month-old infants. Cognitive Psychology, $29,257-302$.

Wisniewski, E., Lamb, C., \& Middleton, E. (2003). On the conceptual basis for the count and mass noun distinction. Language \& Cognitive Processes, 18, 583-624.

Woodward, A., Markman, E., \& Fitzsimmons, C. M. (1994). Rapid word learning in 13- and 18-month-olds. Developmental Psychology, 30 , $553-566$.

Xu, F. (1997). From lot's wife to a pillar of salt: Evidence that physical object is a sortal concept. Mind and Language, 12, 365-392.

Yoshida, H., \& Smith, L. B. (2003a). Correlations, concepts and crosslinguistic differences. Developmental Science, 6, 30-34.

Yoshida, H., \& Smith, L. B. (2003b). Known and novel noun extensions: Attention at two levels of abstraction. Child Development, 74, 564-577.

Yoshida, H., \& Smith, L. B. (2003c). Shifting ontological boundaries: How Japanese- and English-speaking children generalize names for animals and artifacts. Developmental Science, 6, 1-17.

Yoshida, H., \& Smith, L. B. (in press). Linguistic cues enhance the learning of perceptual cues. Psychological Science. 


\section{Appendix A}

\section{Network Update Functions}

The activation update rule used was the rule of the interactive activation and competition model (McClelland \& Rumelhart, 1986).

$$
\text { If } h_{i}^{t}>0 \text {, }
$$

$$
\Delta a_{i}^{t}=h_{i}^{t}\left[a_{i}^{\max }-\left(a_{i}^{t-1}-D_{i} a_{i}^{t-1}\right)\right]
$$

else

$$
\Delta a_{i}^{t}=h_{i}^{t}\left[\left(a_{i}^{t-1}-D_{i} a_{i}^{t-1}\right)-a_{i}^{\min }\right],
$$

where $a_{i}^{t}$ is the activation of unit $i$ at time $t ; h_{i}^{t}$ is the input to unit $i$ at time $t$; and $a_{i}^{\max }, a_{i}^{\min }$, and $D_{i}$ are, respectively, the maximum activation, minimum activation, and decay rate associated with $i$. All units in the network currently have maximum activations of 1 and minimum activations of 0 , and the decay rate was set to .05 for all simulations.
The input to unit $i$ at time $t$ was calculated as

$$
h_{i}^{t}=\sum_{j=1}^{n} a_{j}^{t} \cdot w_{i j},
$$

where $n$ is the number of units in the network and $w_{i j}$ is the weight connecting units $i$ and $j$.

The weight update function used was Contrastive Hebbian Learning (Hopfield, 1982, 1984; Movellan, 1990),

$$
\Delta w_{i j}=\text { phase } * \lambda *\left(a_{i} \cdot a_{j}\right),
$$

where phase is 1 during the clamped phase and -1 during the unclamped or free phase, $\lambda$ is the learning rate or step size of weight change (.001 for all networks), and $a_{i}$ is the activation of unit $i$. 


\section{Appendix B}

Summary of the Architecture, Training, Test Sets, and Comparisons in the Simulations

\begin{tabular}{|c|c|c|c|c|}
\hline Exp. & Architecture & Training set & Testing set & Comparison on test trials \\
\hline 1 & $\begin{array}{l}12 \text { shape units } \\
12 \text { material units } \\
2 \text { solidity units } \\
24 \text { word units } \\
30 \text { hidden layer units }\end{array}$ & $\begin{array}{l}12 \text { solid-shape categories } \\
12 \text { nonsolid-material categories }\end{array}$ & $\begin{array}{l}40 \text { novel instances of each of solid-shape, } \\
\text { nonsolid-shape, solid-material, and } \\
\text { nonsolid-material categories }\end{array}$ & $\begin{array}{l}\text { Solid exemplar: solid shape vs. solid } \\
\text { material } \\
\text { Nonsolid exemplar: nonsolid } \\
\text { material vs. nonsolid shape }\end{array}$ \\
\hline $1 \mathrm{~A}$ & $\begin{array}{l}12 \text { shape units } \\
12 \text { material units } \\
12 \text { size units } \\
12 \text { location units } \\
30 \text { hidden units }\end{array}$ & Same as above & Same as above & Same as above \\
\hline 2 & Same as Exp. 1 & $\begin{array}{l}\text { Patterns in same proportions as } \\
\text { in English-speaking } \\
\text { children's vocabularies }\end{array}$ & Same as Exp. 1 & Same as Exp. 1 \\
\hline 4 & Same as Exp. 1 & Same as Exp. 2 & Same as Exp. 1 & $\begin{array}{l}\text { Cross-solidity sets } \\
\text { Solid exemplar: nonsolid shape } \\
\text { vs. solid material } \\
\text { Nonsolid exemplar: solid material } \\
\text { vs. nonsolid shape }\end{array}$ \\
\hline 6 & Same as Exp. 1 & Same as Exp. 2 & Same as Exp. 1 & $\begin{array}{l}\text { All nonsolid exemplars (simple } \\
\text { shape) } \\
\text { Traditional set: simply shaped } \\
\text { nonsolid shape match vs. } \\
\text { simply shaped nonsolid } \\
\text { material match } \\
\text { Cross-solidity } \\
\text { Simple: simply shaped solid } \\
\text { material match vs. nonsolid } \\
\text { shape match } \\
\text { Complex: complexly shaped solid } \\
\text { material match vs. nonsolid } \\
\text { shape match }\end{array}$ \\
\hline 8 & Same as Exp. 1 & $\begin{array}{l}\text { Patterns in same proportions as } \\
\text { in Japanese-speaking } \\
\text { children's vocabularies } \\
\text { or } \\
\text { Same as in Exp. } 2\end{array}$ & $\begin{array}{l}40 \text { novel instances of each of solid- } \\
\text { complex shape, nonsolid-complex } \\
\text { shape, solid-simple shape, nonsolid- } \\
\text { simple shape, solid-material, and } \\
\text { nonsolid-material categories }\end{array}$ & $\begin{array}{l}\text { Complexly shaped solid exemplar: } \\
\text { solid-complex shape vs. solid- } \\
\text { material shape (randomly } \\
\text { generated shape) } \\
\text { Simply shaped solid exemplar: } \\
\text { solid-simple shape vs. solid- } \\
\text { material shape (randomly } \\
\text { generated shape) } \\
\text { Nonsolid (and thus simply shaped) } \\
\text { exemplar: nonsolid material vs. } \\
\text { nonsolid shape }\end{array}$ \\
\hline 9 & $\begin{array}{l}\text { Same as Exp. } 1+2 \\
\text { syntax units }\end{array}$ & $\begin{array}{l}\text { Same as Exp. } 2+\text { syntax } \\
\text { correlations }\end{array}$ & Same as Exp. 8 & Same as Exp. 8 \\
\hline
\end{tabular}

Note. $\quad$ Exp. $=$ experiment.

Received September 23, 2002

Revision received June 30, 2004

Accepted July 2, 2004 